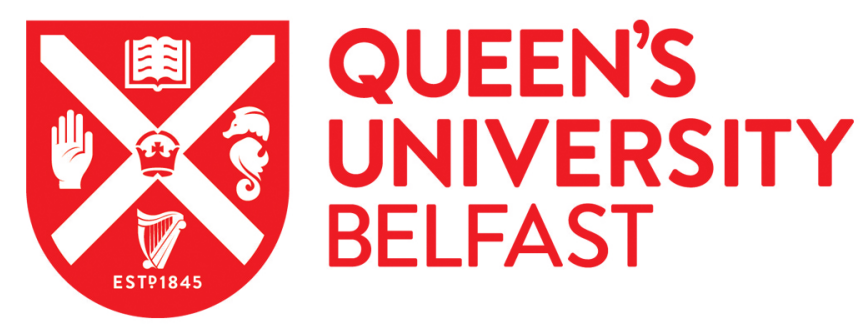

\title{
Mechanochemical Synthesis of Pharmaceutical Cocrystal Suspensions via Hot Melt Extrusion: Enhancing Cocrystal Yield
}

\author{
Li, S., Yu, T., Tian, Y., Lagan, C., Jones, D., \& Andrews, G. (2017). Mechanochemical Synthesis of \\ Pharmaceutical Cocrystal Suspensions via Hot Melt Extrusion: Enhancing Cocrystal Yield. Molecular \\ Pharmaceutics, 15(9), 3741-3754. https://doi.org/10.1021/acs.molpharmaceut.7b00979
}

Published in:

Molecular Pharmaceutics

Document Version:

Peer reviewed version

Queen's University Belfast - Research Portal:

Link to publication record in Queen's University Belfast Research Portal

Publisher rights

(c) 2017 American Chemical Society. This work is made available online in accordance with the publisher's policies. Please refer to any applicable terms of use of the publisher.

\section{General rights}

Copyright for the publications made accessible via the Queen's University Belfast Research Portal is retained by the author(s) and / or other copyright owners and it is a condition of accessing these publications that users recognise and abide by the legal requirements associated with these rights.

Take down policy

The Research Portal is Queen's institutional repository that provides access to Queen's research output. Every effort has been made to ensure that content in the Research Portal does not infringe any person's rights, or applicable UK laws. If you discover content in the Research Portal that you believe breaches copyright or violates any law, please contact openaccess@qub.ac.uk. 


\section{Article}

\section{Mechanochemical Synthesis of Pharmaceutical Cocrystal Suspensions via Hot Melt Extrusion: Enhancing Cocrystal Yield

\author{
Shu Li, Tao Yu, Yiwei Tian, Colette Lagan, David S. Jones, and Gavin P. Andrews
}

Mol. Pharmaceutics, Just Accepted Manuscript • DOI: 10.1021/acs.molpharmaceut.7b00979 • Publication Date (Web): 22 Nov 2017

Downloaded from http://pubs.acs.org on December 7, 2017

\section{Just Accepted}

"Just Accepted" manuscripts have been peer-reviewed and accepted for publication. They are posted online prior to technical editing, formatting for publication and author proofing. The American Chemical Society provides "Just Accepted" as a free service to the research community to expedite the dissemination of scientific material as soon as possible after acceptance. "Just Accepted" manuscripts appear in full in PDF format accompanied by an HTML abstract. "Just Accepted" manuscripts have been fully peer reviewed, but should not be considered the official version of record. They are accessible to all readers and citable by the Digital Object Identifier (DOI®). "Just Accepted" is an optional service offered to authors. Therefore, the "Just Accepted" Web site may not include all articles that will be published in the journal. After a manuscript is technically edited and formatted, it will be removed from the "Just Accepted" Web site and published as an ASAP article. Note that technical editing may introduce minor changes to the manuscript text and/or graphics which could affect content, and all legal disclaimers and ethical guidelines that apply to the journal pertain. ACS cannot be held responsible for errors or consequences arising from the use of information contained in these "Just Accepted" manuscripts. 


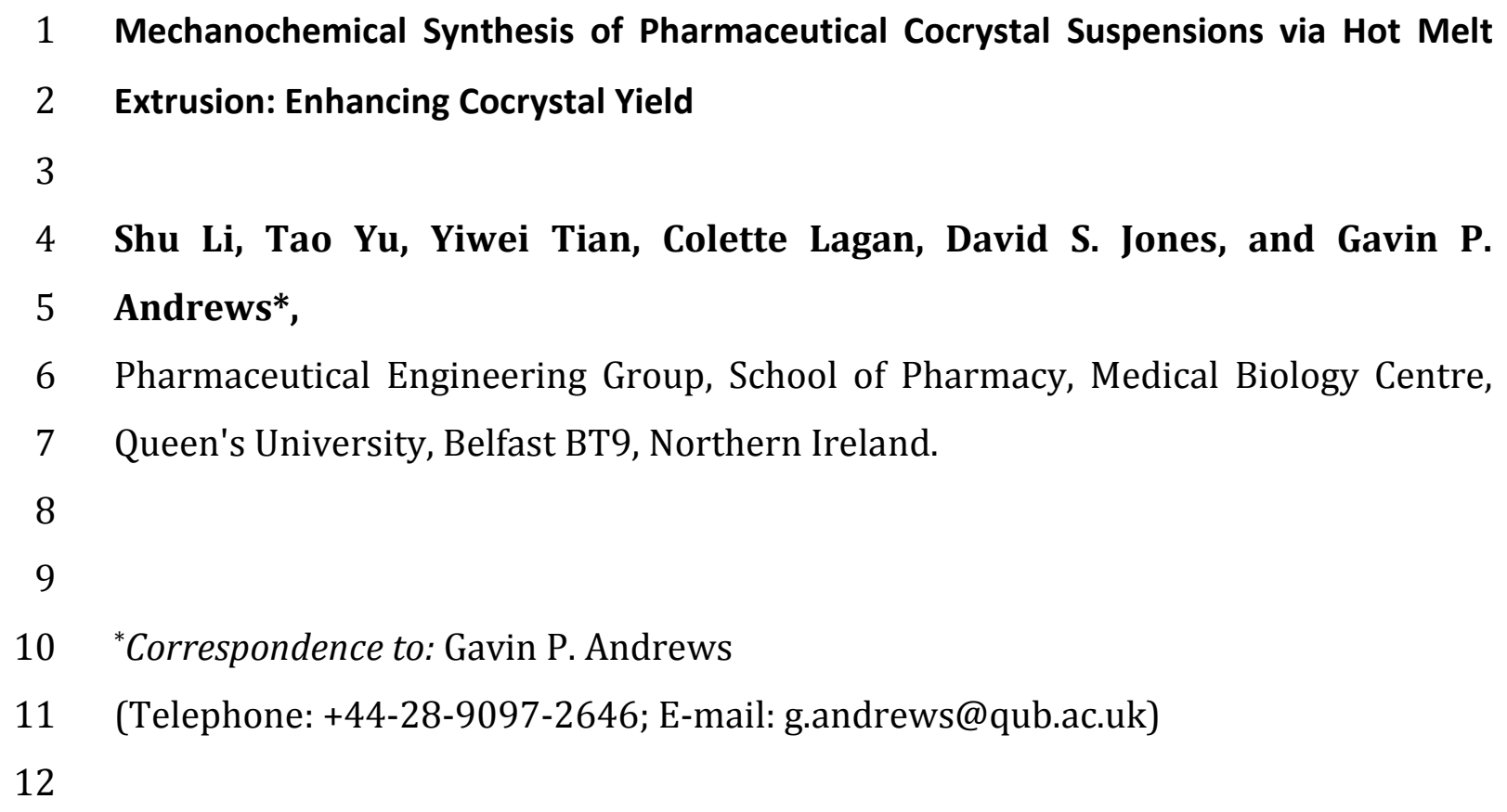




\section{Abstract}

18 Pharmaceutical cocrystals have attracted increasing attention over the past decade

19 as an alternative way to modify the physicochemical properties and hence improve

20 the bioavailability of a drug, without sacrificing thermodynamic stability. Our

21 previous work has demonstrated the viability of in-situ formation of

22 ibuprofen/isonicotinamide cocrystal suspensions within a matrix carrier via a

23 single-step hot-melt extrusion (HME) process. The key aim of the current work is to

24 establish optimised processing conditions to improve cocrystal yield within 25 extruded matrices.

26 The solubility of each individual cocrystal component in the matrix carrier was 27 estimated using two different methods, calculation of Hansen solubility parameters, 28 and Flory-Huggins solution theory using melting point depression measurement, 29 respectively. The latter was found to be more relevant to extrusion cocrystallisation 30 because of the ability to predict miscibility across a range of temperatures. The 31 predictions obtained from the F-H phase diagrams were verified using ternary 32 extrusion processing. Temperatures that promote solubilisation of the parent 33 reagents during processing, and precipitation of the newly formed cocrystal were 34 found to be the most suitable in generating high cocrystal yields. The incorporation 35 of intensive mixing/kneading elements to the screw configuration was also shown 36 to significantly improve the cocrystal yield when utilising a matrix platform. This 37 work has shown that intensive mixing in combination with appropriate temperature 38 selection, can significantly improve the cocrystal yield within a stable and low 39 viscosity carrier during HME processing. Most importantly, this work reports, for 40 the very first time in the literature, the use of the F-H phase diagrams to predict the 41 most appropriate HME processing window to drive higher cocrystal yield. 


\section{INTRODUCTION}

43 Our previous work successfully demonstrated the feasibility of manufacturing 44 (ibuprofen/isonicotinamide) Ibu-IsoNA cocrystals in the presence of a 45 pharmaceutical excipient ${ }^{1}$ using mechanochemical HME processing. From our 46 previous work, it was shown that xylitol was the most appropriate (from the 4 47 selected). Xylitol is a chemically stable pharmaceutical excipient and food additive 48 with a low melting viscosity at specified extrusion temperature ${ }^{2}$. In addition, xylitol 49 was observed to exhibit rapid solidification upon cooling, which was also 50 considered pertinent for the successful extrusion of a cocrystal suspended in the 51 excipient matrix. The extruded cocrystal/xylitol suspensions exhibited further 52 enhanced ibuprofen dissolution rate when compared with the analogous cocrystal 53 extruded devoid of any excipient. However, the final cocrystal yield within the 54 matrix was still unsatisfactory. In our previous study, a small amount of both 55 reagents were rendered amorphous within the matrix during extrusion processing. 56 Subsequently, increased cocrystal yield was observed during storage, particularly 57 when the formulation was placed in a humid environment ${ }^{3}$.

58 It has been indicated in the literature that cocrystallisation by melting or 59 mechanochemical processes may not be as efficient as solution methods, due to 60 limited activity of the reactant molecules ${ }^{4}$. But with the presence of a non61 complementary carrier, both cocrystal reagents could 'dissolve' in the liquefied 62 carrier, resulting in a similar cocrystallisation mechanism to traditional solvent 63 approach, albeit in an increased viscosity environment (due to the viscous nature of 64 the molten excipient). However, since the 'solvent' (matrix carrier) in a HME system 65 will not be removed post processing, the solubility of the cocrystal within the carrier 66 pool should be limited such that precipitation of the product is encouraged. It is 67 therefore obvious that the phase behaviour between individual cocrystal 68 components (including both the reagents and the product of cocrystallisation) and 69 the matrix carrier may significantly influence the extent of cocrystal conversion. A 70 carrier melt pool 'solvent' in which the reagents and the cocrystal show such 71 distinctive solubility differences, or a temperature that drives a solubility difference 
72 between reagents and cocrystal within a given carrier could be used to enhance the 73 cocrystal yield. Therefore, this work is aimed at assessing the solubility of the 74 cocrystal components in a given matrix as well as investigating how HME processing 75 temperature settings may influence solubility and correlation to cocrystal yield. Two 76 different methods have been used to achieve such aims, namely solubility parameter 77 comparison and the adaptation of Flory-Huggins theory using the melting point 78 depression method.

79 It has also been reported in the literature that increased mixing and shear 80 intensity could facilitate cocrystal yield 5 . Therefore, not only can we expect 81 temperature and miscibility to change yield but process parameters such as screw 82 design that is known to alter extrudate properties ${ }^{6}$, and operating efficiency ${ }^{7}$ must 83 be considered. Hence, another important aspect to this research paper is to identify 84 the impact of screw geometry and to rationalize screw configuration for improved 85 cocrystal yield during ternary extrusion processing. Screw configuration designs 86 conveying increased shear intensity were investigated for their influence on 87 cocrystal yield. 


\section{MATERIALS AND METHODOLOGY} Materials

90 Ibuprofen, isonicotinamide, and xylitol were purchased from Sigma-Aldrich (St.

91 Louis, MO, USA). All other chemical reagents used were of analytical grade.

\section{Calculating solubility parameters}

94 The use of solubility parameters to predict the miscibility and/or compatibility of 95 pharmaceutical materials has been extensively reported. Such information is often 96 used to set criteria for excipient selection in formulation design ${ }^{8-10}$, or for coformer 97 selection in cocrystal screening11,12. Estimation of miscibility between any two 98 components (Ibu vs. IsoNA, Ibu vs. xylitol, IsoNA vs. xylitol and, the 1:1 Ibu-IsoNA 99 cocrystal vs. xylitol) within the cocrystal suspension system was performed by 100 comparing the total solubility parameter $\left(\delta_{\mathbf{t}}\right)$ for each individual component. $\delta_{\mathbf{t}}$ was 101 calculated using the Hansen partial solubility parameters $\left(\delta_{\mathbf{d}}, \delta_{\mathbf{p}} \& \delta_{\mathbf{h}}\right)$ based on the 102 group contribution method of Fedors ${ }^{13}$ and Van Krevelen-Hoftyzer ${ }^{14,15}$ :

$$
\delta_{\mathrm{d}}=\frac{\sum \mathrm{F}_{\mathrm{di}}}{\mathrm{V}}, \quad \delta_{\mathrm{p}}=\frac{\sqrt{\sum \mathrm{F}_{\mathrm{pi}}^{2}}}{\mathrm{~V}}, \quad \delta_{\mathrm{h}}=\sqrt{\frac{\sum \mathrm{E}_{\mathrm{i}}}{\mathrm{V}}}
$$

Equation 1

$$
\delta^{2}=\delta_{\mathrm{D}}^{2}+\delta_{\mathrm{P}}^{2}+\delta_{\mathrm{H}}^{2}
$$

108 Where $F_{\text {di }}$ is the group dispersion component contributing to the disperse forces $\delta_{\mathbf{d}}$, $109 \mathrm{~F}_{\mathrm{pi}}$ the plane symmetry factor giving polar group forces $\delta_{\mathbf{p}}, \mathrm{E}_{\mathrm{i}}$ the group contribution 110 to hydrogen bonding energy and V the molar volume from Hildebrand analysis.

\section{Sample preparation for melting point depression measurements}

113 All substances were desiccated over silica gel to guarantee minimum moisture

114 content prior to use. The reference cocrystal was grown via slow evaporation from a 115 methanol solution. The conversion from Ibu and IsoNA to their equimolar cocrystal 116 was confirmed by thermal analysis, powder x-ray diffraction and spectroscopy as 117 described in our previous work ${ }^{1}$. Binary physical mixtures containing compound A 
118 (the reference cocrystal or individual parent reagent, respectively), and compound 119 B (the matrix carrier xylitol) were prepared, using a mortar and pestle, at 120 predetermined compositions $(100 \%, 95 \%, 90 \%, 85 \%, 80 \%, 75 \%$ and $70 \% \mathrm{w} / \mathrm{w}$ of

121 compound A) in triplicate. The resulting mixed powders were desiccated until 122 further use.

123

\section{Differential Scanning Calorimetry (DSC)}

125 Melting point depression experiments for the binary physical mixtures were 126 performed on a model DSC 8000 power compensation differential scanning 127 calorimeter (Perkin-Elmer, Windsor, Berkshire, UK). The machine was calibrated, at 128 the respective heating rates, for melting point and heat of fusion using indium and 129 zinc, respectively, prior to the experiments. Both the reference and sample 130 chambers were purged with dry nitrogen at a flow rate of $40 \mathrm{~mL} / \mathrm{min}$ to maintain an 131 inert atmosphere. 3-5mg of sample was accurately weighed to aluminium pans and 132 crimped using an aluminium pan lid. The crimped pan set was then subjected to a 133 thermal ramp at $1^{\circ} \mathrm{C} / \mathrm{min}$ from $80^{\circ} \mathrm{C}$ to $135^{\circ} \mathrm{C}$. For samples containing IsoNA, a $134200^{\circ} \mathrm{C} / \mathrm{min}$ heating rate and $40 \mathrm{~mL} / \mathrm{min}$ purged helium were used to avoid 135 compositional variations associated with IsoNA degradation at low heating rates 136 (heating rate dependent weight loss observed in TGA analysis, data submitted in the 137 supplementary material). Samples containing IsoNA were subjected to a 30-minute 138 isothermal treatment at $100^{\circ} \mathrm{C}$ prior to the DSC ramping over a range from $-60^{\circ} \mathrm{C}$ to $139230^{\circ} \mathrm{C}$. Such a pre-treatment was employed to provide an extended period so that 140 any inadequate solubilisation caused by the fast heating ${ }^{16}$ might be compensated. $141100^{\circ} \mathrm{C}$ was chosen as the isothermal temperature as it was above the melting of 142 xylitol, yet below the onset of significant IsoNA weight loss $\left(120^{\circ} \mathrm{C}\right.$ if heated at $1431^{\circ} \mathrm{C} / \mathrm{min}$, data shown in supplementary material). The determination of the 144 isothermal time, on the other hand, was based on whether stabilised depression of $145 \Delta \mathrm{H}_{(\mathrm{IsoNA})}$ was attainable during preliminary screening (data included in 146 supplementary document). All values of melting points used in this study were the 147 end temperature of the melting event. 


\section{Powder X-ray Diffraction (PXRD)}

150 PXRD was performed using a MiniFlex II desktop powder X-ray diffractometer 151 (Rigaku Corporation, Kent, England). The machine is equipped with $\mathrm{Cu}$ K $\alpha$ radiation, 152 at a voltage of $30 \mathrm{kV}$ and a current of $15 \mathrm{~mA}$. The scanning was conducted across a $1532 \Theta$ scanning range from $1.5-40^{\circ}$ at $2.0^{\circ} / \mathrm{min}$ and a sample width of $0.03^{\circ}$. The peak 154 area underneath the cocrystal characteristic peak at $3.3^{\circ} 2 \Theta$ for each sample was 155 used to determine the cocrystal yield ${ }^{17}$. A series of physical mixtures containing the 156 reference cocrystal and xylitol at 10 different cocrystal loadings, 10, 20, 30, 40, 50, $15760,70,80,90 \& 100 \% \mathrm{w} / \mathrm{w}$, respectively, were prepared through gentle grinding. 158 The blended samples were placed into a top-fill glass sample holder $(0.2 \mathrm{~mm}$ well 159 depth) for analysis. The peak area was calculated using Integral Int. Calculation 160 software (Integral analysis for windows, Version 6.0, Rigaku Corporation) with 161 manual background subtraction and an integration region set between $162\left[2.400 \sim 4.050^{\circ} 2 \theta\right]$. A calibration curve, $\left(R^{2}=0.998\right)$ was constructed using linear 163 regression between the average peak area and cocrystal concentration in the blends. 164 The calibration curve was validated for linearity, accuracy, precision, LoD and LoQ 165 according to the methods recommended in the ICH guidelines ${ }^{18}$. The results from 166 the construction and validation of the quantitative PXRD analysis were published as 167 supplementary data for our previous work ${ }^{1}$.

Determination of cocrystal yield

170 With the standard calibration curve established and validated, the cocrystal yield in 171 the melt-extruded suspensions can be calculated as follows:

172

$$
\text { Cocrystal yield }(\%)=\frac{\text { Measured cocrystal concentration }(\%)}{\text { Theoretical fully converted content }(\%)} \times 100 \%
$$

173

174 Where the theoretical fully converted content in this work was $50 \%$ as xylitol 175 loading was kept constant at $50 \mathrm{wt} \%$ for all cases.

176 


\section{Hot-melt extrusion (HME)}

178 Hot-melt extrusion was conducted using a co-rotating 20:1 twin screw Rondol 179 Microlab 10mm compounder (Rondol Technology Ltd., Staffordshire, UK) equipped 180 with a force feeder and an open end. Raw materials were accurately weighed, 181 pestle-mixed in a ceramic mortar, and subsequently fed at a fixed rate of 20rpm 182 using the auxiliary single-screw force feeder. A screw speed of 10rpm was used for 183 the main extruder in all extrusion experiments. Table 1 illustrates the temperature 184 profile (TP) settings used in this work. In particular, the temperature for the feeding 185 zone (Zone 0) for all formulations was kept constant at $60^{\circ} \mathrm{C}$ to allow adequate solid 186 conveying from the feed hopper. The following Zone 1 was designed as the melting 187 zone with temperature set at $80^{\circ} \mathrm{C}, 92^{\circ} \mathrm{C}, 100^{\circ} \mathrm{C}, 110^{\circ} \mathrm{C}$ and $115^{\circ} \mathrm{C}$, respectively. To 188 ensure the production of a solid extrudate rather than a melt pool at the barrel exit, 189 Zone 2 \& Zone 3 were designed as cooling zones and set with gradually decreasing 190 temperatures (Table 1).

191 As detailed in Table 2, three different screw configuration profiles (SCP) were 192 applied to modify the mixing and shear intensities and to understand the link to 193 cocrystal yield during HME. SCP1 was assembled using conveying elements only. 194 SCP2 incorporated two kneading regions each configured as a $90^{\circ}$ neutral-staggered 195 kneading block sandwiched by two $60^{\circ}$ forward-staggered kneading blocks. In SCP3, 196 additional kneading blocks were included in each kneading region to provide 197 further enhanced shearing within the melt stream.

198 Extrusion was repeated in triplcate for each formulation and the average 199 residence time and system torque were recorded in Table 3. The resulting 200 extrudates were collected directly from the open barrel end and subsequently 201 pulverized using a mortar and pestle once cooled. The powdered samples were 202 sieved through a $212 \mu \mathrm{m}$ mesh sieve (Scientific Laboratory Supplies Ltd, 203 Nottingham) and then desiccated in non-humid environment prior to further 204 analysis. Note that all formulations extruded in this work contained a fixed xylitol 205 content of $50 \% \mathrm{w} / \mathrm{w}$. 


\section{Statistical analysis}

208 Statistical analyses were conducted using GraphPad Prism one-way analysis of 209 variance with Tukey's multiple comparison tests. The confidence interval was set 210 automatically as $95 \%$. The computed statistics provide a significance value $(\mathrm{P})$ for 211 each individual comparison. A "P" value less than than 0.05 indicates significant 212 difference. 


\section{FLORY-HUGGINS THEORY AND DATA TREATMENT}

214 Flory-Huggins theory is a comprehensively used mathematical theory, developed 215 independently by Flory ${ }^{19,20}$ and Huggins ${ }^{21,22}$, for modelling the thermodynamics of 216 binary polymer-solvent mixtures. In this model, the size disparity between the two 217 components is taken into account when determining the entropy of mixing of a 218 binary system consisting of unequal-sized molecules. The theory assumes 219 hypothetical lattice sites that accommodates either an individual solvent molecule 220 or a polymer chain segment. It is assumed that the macromolecular polymer is 221 equivalently divided into a number of chain segments that each occupies the same 222 volume on a lattice as a solvent molecule in order to utilise the usual expression of 223 entropy of mixing. The free energy of mixing $\Delta \mathrm{G}_{\text {mix }}$ for such a binary system, 224 according to F-H theory, can be expressed using Eq. $3^{23}$ :

225

$$
\frac{\Delta \mathrm{G}_{\mathrm{mix}}}{\mathrm{RT}}=\Phi \ln \Phi+\frac{(1-\Phi)}{\mathrm{m}} \ln (1-\Phi)+\chi \Phi(1-\Phi)
$$

Equation 3

228 Where $\Phi$ is the volume fraction of solvent (the small molecular component), $\mathrm{R}$ is the 229 molar gas constant, $\mathrm{T}$ is the temperature in the Kelvin, $\mathrm{m}$ is the number of polymer 230 chain segments (typically calculated as the ratio of the volume of a polymer chain to 231 that of a solvent molecule), and $\chi$ is the F-H interaction parameter between polymer 232 and solvent molecules ${ }^{19}$. In such an expression, the obtained $\Delta \mathrm{G}_{\mathrm{mix}}$ shows clear 233 composition dependency. Mathematically, the entropy factor (the first two terms on 234 the right-hand side of Eq. 3) of the expression favours mixing by resulting in non235 positive numerical values at all times. The enthalpy factor (the third term), in 236 contrast, shows influence on the mixing preference through the value of $\chi$. For 237 systems exhibiting adhesive bonds favouring miscibility (negative $\chi$ values) and/or 238 no specific bonding between species whatsoever $(\chi=0)$, the $\Delta G_{\text {mix }}$ can remain 239 negative throughout the entire composition range. Whereas for systems that exhibit 240 unfavourable mixing (positive $\chi$ values), the $\Delta \mathrm{G}_{\text {mix }}$ only remains negative until a 241 certain threshold value of $\chi$ and becomes positive at points beyond. 
242 By adapting the concept of the F-H theory, melting point depression theory 24,25 243 may be used in the calculation of $\chi$ value for drug-polymer binaries where the 244 following equations, Eq. 4 and Eq. 5, are applied 16,26-31:

245

246

247

$$
\frac{1}{\mathrm{~T}_{\mathrm{m}}}-\frac{1}{\mathrm{~T}_{\mathrm{m}}^{0}}=-\frac{\mathrm{R}}{\Delta \mathrm{H}}\left[\ln \Phi+\left(1-\frac{1}{\mathrm{~m}}\right)(1-\Phi)+\chi(1-\Phi)^{2}\right]
$$

$$
\chi=\frac{\frac{1}{\mathrm{~T}_{\mathrm{m}}}-\frac{1}{\mathrm{~T}_{\mathrm{m}}^{0}}}{-\frac{\mathrm{R}}{\Delta \mathrm{H}}(1-\Phi)^{2}}-\frac{\ln \Phi}{(1-\Phi)^{2}}-\frac{1-\frac{1}{\mathrm{~m}}}{1-\Phi}
$$

Equation 4

250 Where $\mathrm{T}_{\mathrm{m}}$ is the melting temperature of drug in the binary mixture; $\mathrm{T}_{\mathrm{m}}{ }^{0}$ and $\Delta \mathrm{H}$ are 251 the melting temperature and the enthalpy of fusion, respectively, for the pure drug 252 substance.

253 Based on the expression in Eq. 5, the estimation of the dimensionless, enthalpic 254 energy parameter $\chi$ is related to both temperature and composition. Using 255 experimentally obtained $\mathrm{T}_{\mathrm{m}}$ values for binary mixtures of different drug-polymer 256 compositions, the corresponding value of $\chi$ can be calculated. In practice, however, 257 the measurement of $\mathrm{T}_{\mathrm{m}}$ is always rendered unfeasible at low drug loadings. Ability 258 to extrapolate the value of $\chi$ beyond experimentally feasible temperatures is then 259 crucial to the establishment of phase behaviour throughout the entire composition 260 range. In the current literature, a first order relationship between $\chi$ and the 261 reciprocal temperature (Eq. 6) has been commonly used for such purposes ${ }^{23,29}$ :

$$
\chi=A+\frac{B}{T}
$$

Equation 6

264 Constants A and B can be fitted using the experimental data, allowing the free 265 energy of mixing expression to be become:

266

$$
\frac{\Delta \mathrm{G}_{\mathrm{mix}}}{\mathrm{RT}}=\Phi \ln \Phi+\frac{1-\Phi}{\mathrm{m}} \ln (1-\Phi)+\Phi(1-\Phi)\left(\mathrm{A}+\frac{\mathrm{B}}{\mathrm{T}}\right)
$$


269 Additionally, by setting the second derivative of the $\Delta \mathrm{G}_{\text {mix }}$ to Zero, one can 270 achieve Eq. 8 to determine the position of the spinodal curve ${ }^{32}$, which depicts the 271 boundary of phase separation in the solid state:

272

273

$$
\mathrm{T}_{\mathrm{s}}=\frac{2 \mathrm{~B}}{\frac{1}{\phi}+\frac{1}{\mathrm{~m}(1-\phi)}-2 \mathrm{~A}}
$$

Equation 8

15

16

17 


\section{RESULTS AND DISCUSSION}

276 Cocrystallisation using a conventional solution approach is generally conducted in 277 ternary systems consisting of a conformer, drug and a solvent or a mixture of 278 solvents. Solubility information between individual cocrystal components and the 279 solvent is fundamental in establishing phase diagrams that are generally used to 280 describe the conditions needed for cocrystal formation ${ }^{33-37}$. Moreover, it has been 281 shown that coformer solubility in solvent during liquid-asssited mechanochemical 282 synthesis plays an important role, influencing cocrystallisation efficiency $4,38-43$.

283 In our work, we consider the synthesis of a cocrystal in a molten carrier to be 284 similar to that in the presence of a traditional organic solvent. The liquefied carrier 285 serves as a viscous solvent to accommodate the parent reagents, hence providing 286 increased degrees of freedom for hetero-molecular collision and thus improved 287 cocrystallisation efficiency. In previous research, it has been suggested that the 288 solvent should not be capable of forming secondary interactions with any cocrystal 289 reagents such that the interactions between the reagents are still favoured. In 290 respect of HME, processing conditions may be controlled in order to enhance 291 interaction between cocrystal reagents and limit reaction with meltable carrier 292 'solvent' 44,45 . In addition, we should also remember that unlike a traditional organic 293 solvent used for either solvent-based cocrystallisation or liquid-assisted grinding, 294 the molten carrier used during HME will not evaporate after processing, but rather 295 remains as a matrix. As such the pharmaceutical excipient used as a matrix carrier 296 should be suitable to meet the end use of the drug product. In particular, the 297 dispersed cocrystal phase must have limited solubility in the matrix carrier at 298 decreased temperature when the formulation exits the extruder in the solid-state. 299 Without this condition being met there may be a high probability that an amorphous 300 dispersion of individual coformer molecules, rather than a cocrystal suspension 301 would form within the carrier ${ }^{46}$.

302 To aid understanding of this hypothesis, an illustration showing the preparation 303 of cocrystal suspensions in a single-step HME is provided in Figure 1. Using this 304 method, the manufacture of cocrystal suspensions may be regarded as a sequence of 
305 events in the direction of melt flow. Ideally, the ternary physical mixture is fed into 306 the extruder feed zone as mixed solids. The mixed solids are then conveyed to a 307 melting zone where a melt pool forms. The presence of such a melt pool provides a 308 medium for cocrystallisation and additionally may also significantly improve 309 reaction kinetics due to the increased mobility within the molten matrix ${ }^{47}$. The 310 processing temperature in the following zone should be decreased sufficiently to 311 assist precipitation of the newly formed cocrystals from the carrier melt, whilst the 312 final zone ought to be designed with a further decreased temperature to ensure 313 solidification of the carrier, and thus, formation of an intimately mixed cocrystal 314 suspension. Consequently, it is extremely important to understand solubility 315 behaviour and miscibility of individual cocrystal components and the carrier.

316 Interestingly, in the scenario presented, solubility and component miscibility must

317 be considered both in the melt and also in the solid-state. In this work, we present 318 two extensively reported methods for the purposes of advancing our understanding 319 of this complex cocrystal manufacturing process.

\section{Solubility Parameters}

322 The Hansen solubility parameters (HSP) calculated using the group contribution 323 method has enabled the prediction of solid-solid solubility of pharmaceutical 324 relevant materials 8,9 . For drug-excipient combinations, the Forster predictions 325 consider a $\Delta \delta_{\mathbf{t}}<7.0 \mathrm{MPa}^{1 / 2}$ indicative of significant miscibility and formation of glass 326 solutions during melt-extrusion, whereas a $\Delta \delta_{t}>10.0 \mathrm{MPa}^{1 / 2}$ denotes a lack of 327 miscibility and limited ability to form glass solutions ${ }^{10}$. Later, Velaga et al., explored 328 the correlation between solubility parameter calculations, predicted drug-coformer 329 miscibility and the feasibility of cocrystallisation ${ }^{11}$. In this important work, it was 330 shown that those systems capable of successfully forming cocrystals were 331 manufactured from systems that were miscible. The authors therefore concluded 332 that drug-coformer miscibility was necessary in order to attain cocrystallisation, 333 and that the use of solubility parameters was an efficient method for screening 334 coformer candidates. 
In the light of the above, our current study considered that Ibu and IsoNA should show significant good miscibility thus encouraging cocrystallisation. Moreover, our selected carrier (Xylitol) should exhibit limited miscibility with the formed cocrystal. Within this investigation our hypothesis is that dissolution of the parent reagents within molten xylitol could increase activity of the reactants hence increasing interspecies collision, leading to improved cocrystal yield. However, interaction 341 between xylitol and each individual cocrystal reagent must not surpass that 342 between the parent reagents.

343 The solubility parameter values for parent reagents, cocrysal and xylitol were 344 calculated and compared. Table 4 gives a list of the identified component group 345 contributions and the calculated total solubility parameter for each compound. The 346 subsequent calculation of the total solubility parameter difference $\Delta \delta_{t}$, is as follows: $347 \Delta \delta_{\mathrm{t}}(\mathrm{Ibu} / \mathrm{IsoNA})$ is $1.95 \mathrm{MPa}^{1 / 2}, \Delta \delta_{\mathrm{t}}$ (Ibu/xylitol) and $\Delta \delta_{\mathrm{t}}$ (IsoNA/xylitol) are 13.56 $348 \mathrm{MPa}^{1 / 2}$ and $11.61 \mathrm{MPa}^{1 / 2}$, respectively, whilst $\Delta \delta_{\mathbf{t}}$ (cocrystal \& xylitol) is 13.74 $349 \mathrm{MPa}^{1 / 2}$. According to Forster et al., the $\Delta \delta_{\mathbf{t}}$ values obtained confirm, theoretically, 350 that Ibu and IsoNA are strongly miscible, whilst xylitol is less miscible with both the 351 cocrystal reagents and the respective equimolar cocrystal. The predicted 352 immiscibility between xylitol and the Ibu-IsoNA cocrystal may be beneficial in 353 precipitation of cocrystal product from the excipient following extrusion. Unlike 354 cocrystallisation using conventional solvents in which cocrystal production is 355 facilitated through evaporation of solvent, formulating a cocrystal suspension via 356 HME is fundamentally different in the sense that the excipient carrier solidifies 357 rather than evaporates after processing. Therefore precipitation, in such cases, can 358 only occur when the newly formed cocrystal exhibits a significantly reduced 359 miscibility/solubility with the carrier excipient. Interestingly, the limited miscibility 360 predicted using the solubility parameter approach between xylitol and each 361 individual parent reagent (Ibu and IsoNA), presents a complex scenario for 362 evaluation of such a system. In such cases the limited miscibility may render the 363 presence of xylitol a physical hindrance thus preventing reaction of the parent 364 reagents and formation of cocrystal. However, it must be noted that the calculated 365 solubility parameter values do not take in to account the shearing forces and 
366 temperatures involved during extrusion, which may promote reaction of the two 367 components. Moreover, miscibility predictions are very much dependent upon 368 temperature and the relationship to the solubility parameter. Comprehensive group 369 contribution information throughout a greater temperature scale other than $37025^{\circ} \mathrm{C}^{48,49}$ is still lacking, rendering the HSP method somewhat less useful while 371 assessing the mixing behaviour at significantly elevated temperatures during the 372 HME processing. Furthermore, in our previous work describing the manufacture of 373 cocrystals via HME we indeed demonstrated the successful formation of an 374 equimolar Ibu-IsoNA cocrystal from a ternary mixture with xylitol ${ }^{1}$.

375

376

\section{Melting Point Depression Measurement and Construction of Phase Diagrams}

377 The use of Flory-Huggins theory in combination with melting point depression

378 measurements, has recently been utilised to better understand the relationship 379 between binary mixing behaviour and temperature. In particular, F-H theory has 380 been shown to be useful in determining the impact of processing temperature on 381 the miscibility of components during melt extrusion $16,29,31,50$. Although F-H theory 382 has been used for polymeric and other viscous systems, the high melt or rubber 383 viscosity provide a kinetic barrier to dissolution. The hindered mobility in these 384 systems often leads to an underestimation of solubility. In this current study 385 involving xylitol, a small molecular weight sugar alcohol, the similarity of molecular 386 weight between components and the low viscosity of xylitol reduce the likelihood of 387 underestimation. In the original F-H theory, a hypothetical 'lattice' in space is 388 assumed as the spatial volume that a polymer chain segment or a solvent molecule 389 occupies. The fact that both the solute and solvent in this case are small molecules 390 reduces the extent of dissimilarity in molecular size. In our consideration, instead of 391 assuming a hypothetical space, we took into account the size dissimilarity directly 392 by calculating the volume ratio of a carrier molecule to a drug molecule using their 393 true densities and respective molecular weights (Table 5).

394 Ibuprofen, has a lower melting temperature $\left(76.86 \pm 0.08^{\circ} \mathrm{C}\right)$ relative to xylitol $395\left(95.32 \pm 0.14^{\circ} \mathrm{C}\right)$ and thus it is reasonable to assess xylitol solubility in molten 
396 ibuprofen. It is also worth noting that when determining the mixing between xylitol 397 and ibuprofen, xylitol is considered the 'solute' while ibuprofen is considered the 398 'solvent'. In the design of the latter melt extrusion experiments, ibuprofen and 399 xylitol were subjected to fusion simultaneously. The true density, $\mathrm{T}_{\mathrm{m}}$ and $\Delta \mathrm{H}$ for IBU, 400 IsoNA, xylitol and the reference cocrystal are listed in Table 5. The melting 401 endotherms are shown in Figure 2 for ibuprofen-xylitol, isonicotinamide-xylitol and 402 cocrystal-xylitol binary systems, respectively. It is worth noting that a DSC heating 403 rate of $1^{\circ} \mathrm{C} / \mathrm{min}$ was applied to both the xylitol/Ibu and cocrystal/xylitol systems, 404 whilst $200^{\circ} \mathrm{C} / \mathrm{min}$ was employed for the IsoNA/xylitol system. This was because 405 IsoNA was discovered to sublime hence undergoing tremendous weight loss before 406 reaching its melting point. Such sublimation was heating rate-dependent, with 407 prominently increased loss of weight if sample was treated with extremely slow 408 heating such as $1^{\circ} \mathrm{C} / \mathrm{min}$, but negligible weight loss if heated using $200^{\circ} \mathrm{C} / \mathrm{min}$ (See 409 Appendix 1 in the supplementary document). Our previous publication has 410 investigated the influence of DSC heating rate upon the construction of drug411 polymer miscibility phase diagram when applying the $\mathrm{FH}$ theory to the melting 412 point depression data ${ }^{16}$. It was shown in that work the use of high DSC heating rates 413 could result in underestimation of the metastable region and inaccurate prediction 414 of a poor miscibility limit. The authors attributed such inaccuracy to inadequate 415 dissolution of molecules from within the drug crystalline lattice into the polymer, 416 when increased DSC heating rates were applied. However, in the current work, the 417 dissolution of IsoNA in molten xylitol does not necessarily suffer from the same 418 extent of time-dependence, since xylitol is a small molecular component with 419 significantly lower melting viscosity. Though that being said, a compensational 420 isotherm was conferred on the IsoNA/xylitol mixtures, prior to the DSC experiments. 421 This isotherm was performed at $100^{\circ} \mathrm{C}$, well below the onset of IsoNA weight loss 422 (Appendix 1 in the supplementary document), but above the melting of xylitol, 423 sufficient to create a melt pool of xylitol for IsoNA to dissolve in. A time frame of 30 424 min was selected from a series of tested periods (15, 30 and $45 \mathrm{~min}$ ) since it 425 provided a stabilised heat of fusion depression (see Appendix 2 in the 426 supplementary document). 
427 Melting depression was evident in each system, for example in the xylitol/Ibu 428 blends, the end point for the xylitol melting was measured to shift gradually from $42995.32 \pm 0.14^{\circ} \mathrm{C}$ to $94.84 \pm 0.07^{\circ} \mathrm{C}$ with gradual increase of the ibuprofen content from $430 \quad 0-30 w t \%$ (Figure 2a, for mean and standard deviation of individual measurement 431 please see Appendix 3 in the supplementary document). Interestingly, although 432 ibuprofen melted at a lower temperature than xylitol, the presence of the latter was 433 observed to depress the melting of ibuprofen from $78.86 \pm 0.08^{\circ} \mathrm{C}$ to $\left(76.44 \pm 0.10^{\circ} \mathrm{C}\right)$. 434 Similarly, increase of the xylitol content in both IsoNA/xylitol and cocrystal/xylitol 435 systems also resulted depression of IsoNA and the cocrystal melting from $436169.79 \pm 1.62^{\circ} \mathrm{C}$ to $150.52 \pm 0.42^{\circ} \mathrm{C}$ (Figure 2b), and from $120.96 \pm 0.06^{\circ} \mathrm{C}$ to $437115.43 \pm 0.07^{\circ} \mathrm{C}$ (Figure 2c), respectively (see Appendices 4 and 5 in the 438 supplementary document for mean and standard deviation of individual 439 measurement). A small endothermic event $\left(141.29 \pm 0.21{ }^{\circ} \mathrm{C}\right)$ was also observed on 440 the pure IsoNA DSC thermogram, shortly prior to the main melting at $169.79 \pm 1.62^{\circ} \mathrm{C}$ 441 (Figure 2b). This corresponds to the thermal behaviour of IsoNA polymorph 2 (CSD 442 refcodes EHOWIH, EHOWIH01) ${ }^{51}$. The small initial endotherm has been previously 443 reported to be the result of solid-solid phase transition into modified forms which 444 would then melt as IsoNA polymorph $2^{52}$.

445 Using the melting depression data, the Flory-Huggins interaction parameter, $\chi$, 446 was calculated for each respect system (across experimentally measurable 447 temperatures) using Eq. 5 and plotted against the corresponding reciprocal of $\mathrm{T}_{\mathrm{m}}$ in 448 Figure 3. Linear regression shows reasonably good fits $(R>0.94)$ for all three 449 systems. As discussed before, although our previous paper has shown the impact of 450 heating rate on the construction of the F-H phase diagrams, and more directly on the 451 values of $\chi^{16}$, such impact was again considered not significant in the current work, 452 due to the limited kinetic hindrance (since all species involved were small 453 molecules) present in the system. Indeed, in Figure $3 b$, negative $\chi$ values were 454 calculated for the IsoNA/xylitol blends with increased negativity when increasing 455 the xylitol content. A negative A value calculated from the linear fit (Eq. 6), in 456 particular, suggests negative Gibb's free energy and favourable mixing between the 
457 two species due to favourable entropic contributions in the system. Interestingly for 458 the xylitol/ibuprofen and the cocrystal/xylitol blends, both $\chi-1 / T$ plots resulted in 459 positive A values instead (Figure 3a and 3c, respectively), suggesting unfavourable 460 mixing and positive Gibb's free energy at the depressed melting temperatures of 461 xylitol and the cocrystal.

462 A plot of $\Delta \mathrm{G}_{\mathrm{mix}} / \mathrm{RT}$ versus compound fraction for each system is shown in Figure 4634 based on Eq. 7, denoting the variation of free energy of mixing as a function of 464 both composition and temperature. It is worth nothing that the temperatures 465 chosen for the calculation of $\Delta \mathrm{G}_{\mathrm{mix}} / \mathrm{RT}$ were the nearest integers to the following 466 criteria: (1) above ibuprofen melting endpoint $-80^{\circ} \mathrm{C}$; (2) onset of xylitol melting $46792^{\circ} \mathrm{C}$; (3) end of xylitol melting $-95^{\circ} \mathrm{C}$; (4) onset of the equimolar Ibu-IsoNA 468 cocrystal $-120^{\circ} \mathrm{C}$; and (5) intermediate temperatures between (3) and (4) $-100^{\circ} \mathrm{C}$, $469110^{\circ} \mathrm{C}$ and $115^{\circ} \mathrm{C}$, respectively. Generally speaking, a negative energy value is 470 indicative of mixing in which attraction forces between species are stronger than 471 that between like molecules promoting the occurrence of a homogeneous phase, 472 whereas a positive energy value signifies immiscibility. The shape of the energy 473 curve at a specific temperature is also informative. A concave curve is often 474 observed in homogeneous phases revealing negative energy values throughout the 475 entire composition range. A convex shape, on the other hand, suggests spontaneous 476 phase separation for all compositions at this temperature. A sigmoidal curve, 477 therefore, is indicative of concentration dependent phase behaviour at the defined 478 temperature.

479 In Figure 4a, it is shown that the energy of mixing between ibuprofen and xylitol 480 is almost zero throughout the entire composition range when the temperature is $48195^{\circ} \mathrm{C}$. Concave energy curves are observed for both $92^{\circ} \mathrm{C}$ and $80^{\circ} \mathrm{C}$, while convex 482 curves are observed from $100^{\circ} \mathrm{C}$ to $120^{\circ} \mathrm{C}$. Such energy diagrams suggest that 483 ibuprofen is less miscible with xylitol when temperature elevates, agreeing with the 484 previously obtained positive $A$ and $\chi$ values. A similar trend is also observed for the 485 cocrystal/xylitol blends in Figure 4c where the energy curve changes from a 486 concave profile at $80^{\circ} \mathrm{C}$ (favourable mixing) to approximating a plateau (majority of 487 the energy values just above zero) when the temperature reaches $115^{\circ} \mathrm{C}$. Further 
488 increase of the temperature to $120^{\circ} \mathrm{C}$ resulted in a convex energy curve, suggesting 489 limited miscibility at such a temperature. The IsoNA/xylitol blends, on the other 490 hand, exhibited concave energy curves from $110-120^{\circ} \mathrm{C}$ (Figure $4 \mathrm{~b}$ ), indicative of 491 complete miscibility between IsoNA and xylitol across the entire composition range 492 at these temperatures. Interestingly, although the energy curve was sigmoidal at $493100^{\circ} \mathrm{C}$, all the values of $\Delta \mathrm{G}_{\text {mix }} / \mathrm{RT}$ were negative, suggesting favoured interspecies 494 mixing at this temperature too.

495 Derived from these energy curves, the thermodynamic (temperature496 composition) binary phase diagrams were constructed for xylitol/ibuprofen, 497 IsoNA/xylitol and the reference cocrystal/xylitol mixtures, respectively, enabling 498 the prediction of binary miscibility as a function of temperature. From Figures $5 \mathrm{a}$ 499 and 5c, it can be seen that a Lower Critical Solution Temperature (LCST) behaviour 500 was evident for both the xylitol/ibuprofen and the cocrystal/xylitol systems, with 501 the calculated critical temperatures to be approximately $95^{\circ} \mathrm{C}$ and $114^{\circ} \mathrm{C}$, 502 respectively. The LCST is the shared minimum of the two F-H theoretical curves, the 503 concave spinodal and binodal lines, respectively. A LCST behaviour denotes 504 complete miscibility in all proportions at temperatures below the LCST. In the 505 previous energy curves, a plateau line was reached at $95^{\circ} \mathrm{C}$ to distinguish the 506 boundary between complete miscibility (concave energy curve) and limited 507 miscibility between xylitol and ibuprofen. A similar plateau was also observed at $508115^{\circ} \mathrm{C}$ to denote complete and limited miscibility boundary between the cocrystal 509 and xylitol. Therefore, the LCST behaviour observed for the two systems agrees with 510 the previous energy curve indications and is suggesting complete miscibility 511 between xylitol and ibuprofen below $95^{\circ} \mathrm{C}$ and that between the cocrystal and xylitol 512 below $114^{\circ} \mathrm{C}$ (or $115^{\circ} \mathrm{C}$ if not taking into account the $1^{\circ} \mathrm{C}$ discrepancy between the 513 LCST and the $\Delta \mathrm{G}_{\text {mix }} / \mathrm{RT}$ plateau), respectively. Such result is most interesting yet 514 counter-intuitive as it is advising complete miscibility between these species at 515 room temperature. Although the existence of an LCST for small molecules is rare, 516 there are previous reports of immiscibility between ibuprofen and xylitol when both 517 compounds are molten ${ }^{9}$. Complete miscibility between the Ibu-IsoNA cocrystal and 518 xylitol, on the other hand, could contradict with the "immiscible with newly formed 
519 cocrystal" criteria for carrier screening in the first place. However, one must 520 remember that all species are in the solid crystalline state at room temperature, 521 hence the likelihood of cocrystal dissolution in the rigid xylitol crystalline lattices 522 would be extremely small due to strong kinetic hindrance.

523 From Figure 5b, an Upper Critical Solution Temperature (UCST) behaviour is 524 observed for the IsoNA/xylitol system with the critical temperature calculated to be $525110^{\circ} \mathrm{C}$. Such result indicates complete IsoNA-xylitol miscibility at temperatures 526 greater than $110^{\circ} \mathrm{C}$, in conformity with the miscibility boundary suggested by the 527 concave $\Delta \mathrm{G}_{\text {mix }} / \mathrm{RT}$ curves in Figure $4 \mathrm{~b}$. However, it is worth noting that the negative $528 \Delta \mathrm{G}_{\mathrm{mix}} / \mathrm{RT}$ values at all proportions on the sigmoidal energy curve at $100^{\circ} \mathrm{C}$ (Figure 529 4b), although signifies thermodynamically favoured mixing, still exhibits a $10^{\circ} \mathrm{C}$ 530 difference than what would be required for ideal mixing (UCST).

531

532 Hot-melt Extrusion with Predetermined Temperature Profiles and Screw Configuration 533 Profiles

534 Phase Diagrams and relevance to Processing

535 From melting depression data previously discussed, ideal mixing would occur below $53695^{\circ} \mathrm{C}$ between ibuprofen and xylitol, however above $110^{\circ} \mathrm{C}$ to ensure (or at least 537 higher than $100^{\circ} \mathrm{C}$ to favour) dissolution of isonicotinamide in the molten xylitol. 538 Moreover, processing should be carried out above $115^{\circ} \mathrm{C}$ to facilitate precipitation of 539 cocrystal product from the xylitol melt (LCST at $114^{\circ} \mathrm{C}$ and all-proportion negative $540 \Delta \mathrm{G}_{\text {mix }} / \mathrm{RT}$ values above $115^{\circ} \mathrm{C}$ ). Using this theoretical framework makes it 541 impossible to identify a processing window that would be ideal for mixing of parent 542 reagents with xylitol whilst encouraging precipitation of the cocrystal product 543 (Figure 6). Despite this, our previous work successfully prepared an equimolar Ibu544 IsoNA cocrystal (albeit with relatively low yield) at $92^{\circ} \mathrm{C}$, the temperature being 545 selected to correspond to the onset melting temperature for xylitol, in a HAAKE 546 Minilab conical twin-screw extruder 1.

547 In order to understand how useful the F-H theory is with respect to manufacture 548 of cocrystal material and in identifying relevant processing windows, temperatures 
549 previously shown to be critical in both the energy curves and T- $\Phi$ phase diagrams $550\left(80,95,100,110\right.$ and $115^{\circ} \mathrm{C}$, respectively) were used for Zone 1 during extrusion. 551 This zone was used as a melt zone for the ternary system, consisting of an equimolar 552 Ibu-IsoNA premix blended with xylitol at a 1:1 mass ratio. The feed zone (Zone 0) 553 upstream to Zone 1 was kept constant at $60^{\circ} \mathrm{C}$ to allow adequate solid conveying. 554 Zones 2 and 3 downstream to the melt Zone 1 were set at gradually cooled 555 temperatures to ensure solidification of the extrudates. The respective temperature 556 profiles (TPs) were named as TP1-TP5 (Table 1), respectively.

557 Extrusion trials 1-5 were conducted with a full set of conveying screw elements 558 (SCP1). As shown in Figure 7, when SCP1 was used, temperature profiles TP1, TP2 559 and TP3 resulted in low but gradually increasing cocrystal yields $(9.0 \pm 0.2 \%$, $5609.7 \pm 1.2 \%$ and, $12.6 \pm 2.0 \%$ respectively). TP4 gave the highest yield of $31.4 \pm 1.9 \%$ 561 with TP5 showing a small but significant decrease in yield to $22.1 \pm 1.2 \%$. 562 Interestingly, according to previous phase diagram predictions, although $110^{\circ} \mathrm{C}$ 563 (TP4 melting zone temperature) is favouring IsoNA dissolution in the xylitol melt, 564 this temperature is unfavourable for miscibility between xylitol and ibuprofen. If 565 based on the original hypothesis that dissolution of both parent coformers is 566 required for cocrystallisation, TP4 should result in just as low cocrystal yield as TP1 567 and TP2. However, it was clearly evident that IsoNA/xylitol miscibility (as with TP4) 568 played a more dominant role in driving cocrystallisation to higher yield, whereas 569 the influence of xylitol/ibuprofen miscibility (as with TPs1-3) was much less 570 pronounced. This is probably because when dissolved in the xylitol melt, the 571 conformational freedom open to IsoNA molecules is significantly enhanced at 572 various interfaces, so there is greater probability for molecular collision ${ }^{53}$. With 573 regard to ibuprofen, on the other hand, melting of the drug occurred at all 574 investigated processing temperatures, and thus, there was already a high ibuprofen 575 molecular mobility 5 . It also appeared that there was no significant impact on 576 cocrystal yield whether molten ibuprofen was miscible (TP1) or immiscible (TP2 \& 577 TP3) with xylitol. In fact, FTIR analysis within our preliminary investigations 578 showed no evidence of molecular interaction between ibuprofen and xylitol 579 molecules in a mixture prepared by melting followed with quench cooling. This 
580 would have created almost a competing environment between the molten ibuprofen 581 and the molten xylitol to interact with the dissolved IsoNA molecules. Therefore, it 582 would be crucial that the interactions responsible for IsoNA-xylitol miscibility are 583 not substantially stronger than that responsible for the ibuprofen-IsoNA 584 cocrystallisation.

585 It is also worth noting that when using $100^{\circ} \mathrm{C}$ as the melting zone temperature 586 (TP3), although the energy curve predicted negative $\Delta \mathrm{G}_{\text {mix }}$ at all compositions 587 (suggesting favoured IsoNA/xylitol mixing), the cocrystal conversion yielded from 588 TP3 $(12.6 \pm 2.0 \%)$ was only $1 / 3$ of that from TP4 $(31.4 \pm 1.9 \%)$. Since the temperature 589 setting $\left(110^{\circ} \mathrm{C}\right)$ used in TP4 was the UCST in the binary system denoting boundary 590 of complete IsoNA/xylitol miscibility, significant cocrystal yield increasing from TP4 591 is suggesting that the critical temperature prediction using the T- $\phi$ phase diagram 592 may offer more guidance in selecting processing temperature rather than a simple 593 reliance on temperatures when $\Delta \mathrm{G}_{\text {mix }}$ is negative.

594 The phase diagrams also suggested cocrystal/xylitol phase separation at 595 temperatures higher than $114^{\circ} \mathrm{C}$ (the LCST). Since Zone 2 and Zone 3 were set at 596 gradually decreasing temperatures to allow sufficient solidification for xylitol, it is 597 presumed that the Ibu-IsoNA cocrystal would precipitate out from the xylitol melt in 598 the melting Zone 1, where phase separation is thermodynamically favoured. 599 Moreover, the precipitation of the cocrystal from xylitol might drive further 600 dissolution of IsoNA into the molten carrier. Therefore, it was anticipated that TP5, 601 having $115^{\circ} \mathrm{C}$ set as the melting zone temperature, should result in further increased 602 cocrystal yield than TP4. However, with full conveying screw geometry (SCP1), the 603 resulted yield from TP5 was only $2 / 3(22.1 \pm 1.2 \%)$ of that from TP4. Although such 604 result seems to suggest inaccuracy of the cocrystal/xylitol phase diagram 605 predictions, one should remember that, in extrusion, it is common to have a higher 606 melt temperature than the set values. Such temperature variations are typically the 607 result of local temperature fluctuations derived from the frictional heat generated 608 during shearing. In the current work, the LCST denoting cocrystal/xylitol phase 609 separation $\left(114^{\circ} \mathrm{C}\right)$ was too close to the onset of cocrystal melting $\left(120.21 \pm 0.26^{\circ} \mathrm{C}\right)$. 
610 With increasing cocrystal yield and hence increased amount of solid content, the 611 frictional heat accumulation increases, and so does the regional temperature. Once 612 the local temperature rise approaches close to the cocrystal melting temperature, 613 the newly formed cocrystal would start to melt. If the cocrystal melting overweighs 614 their increased formation, a reduced overall yield (relative to TP4) would be 615 measured. Therefore, while using the T- $\phi$ phase diagram to predict the HME 616 processing window for cocrystal suspensions, the influence of the mechanical 617 shearing upon phase transition, such as melting, of the cocrystal components should 618 not be ignored.

\section{Screw Configuration and Cocrystal Yield}

621 When extruding the binary mixture of a coformer pair, increased mixing intensity 622 has been reported to contribute to improved cocrystal yield 5 . It is of interest to see 623 how varying the level of mixing could impact upon cocrystal yield, particularly in 624 this complex ternary system consisting of a coformer pair and a matrix carrier 625 (xylitol). More importantly, it is interesting to understand how this information can 626 be used to design a screw configuration that drives improved cocrystallisation.

627 Consequently, the ternary blend consisting of premixed equimolar Ibu-IsoNA and 628 xylitol at 50:50 weight ratio was subjected to two additional screw configurations 629 SCP2 and SCP3, respectively. Initially, both the SCP2 and SCP3 contain forward 630 conveying elements for the feed Zone 0 to ensure material transport (Figure 8a). To 631 facilitate mixing and the subsequent formation of cocrystals, kneading blocks were 632 introduced to Zone 1 . The kneading block designs are the most commonly used 633 mixing element among numerous element designs for co-rotating twin-screw 634 extruders. This is due to their mixing efficiency and self-wiping nature. A block of 635 kneading discs, are usually described as stagger angle between two adjacent discs, 636 number of discs in one block and overall length of the block. The kneading blocks 637 used in this study include 60/4/10 elements $\left(60^{\circ}\right.$ stagger angle with 4 discs forming 638 a 10mm long block), and 90/4/10 elements for SCP2 and SCP3, respectively. 
Generally speaking, for kneading blocks that are arranged in a forward conveying 640 direction, a smaller stagger angle provides more positive melt flow (pumping 641 capability). A kneading element with a perpendicular disc design (a $90^{\circ}$ stagger 642 angle) is called a neutral kneading block that has neither forward nor reverse 643 conveying attributes. The conveying of materials through a neutral block is entirely 644 dependent upon forward conveying elements upstream the block to compensate for 645 the lack of conveying action in the neutral kneading region ${ }^{54,55}$. Therefore, an 646 increased number of neutral kneading blocks usually results in increased extrusion 647 residence time owing to reduced forward conveying capacity. Figure $8 \mathrm{~b}$ shows the 648 detailed kneading block arrangement employed in SCP2. A 90/4/10 block was 649 sandwiched between two 60/4/10 blocks. A gradually increasing then 'phase out' 650 sequence is usually deemed necessary to provide a transition between complete 651 forward flow and complete stagnation so that local pressure within the neutral 652 kneading region does not increase dramatically. The same sequence was used both 653 in Zone 1 and between Zone 2 and Zone 3 in (Table 2, SCP2). It is worth noting that 654 two separate kneading regions were used to prevent excessive stagnation of the 655 melt flow. The measured cocrystal yields are shown in Table 3, (SCP 2, formulations 656 6-10). In general, the percent cocrystal yield was dependent upon Zone 1 657 temperature setting, with $13.8 \pm 0.3 \%, 13.9 \pm 0.1 \%, 20.6 \pm 0.4 \%$ and $44.2 \pm 0.3 \%$ 658 measured for TP1-4, respectively, and $30.5 \pm 0.8 \%$ for TP5. The incorporation of 659 kneading sections was evidently in favour of increased cocrystal yield. Moreover, 660 the overall correlation between the cocrystal yield and the TP settings was still in 661 agreement with what had been previously achieved using full convey screw 662 configuration (SCP1). TP5, although anticipated to offer further increased cocrystal 663 yield according to the T- $\phi$ phase diagram predictions, resulted in a reduced yield 664 than TP4. This, again, emphasises the importance of mechanical input during HME, 665 its impact upon local temperature fluctuation and phase transition (i.e. melting) of 666 the produced cocrystal.

667 In addition, the stagger angle between adjacent kneading discs controls the inter668 disk leakage, which dictates whether the kneading unit is more prone to dispersive 
669 or distributive mixing (Figure 9). Kneading elements that show a smaller stagger 670 angle or broader kneading disc thickness typically contribute to increased 671 dispersive mixing, whereas those with larger stagger angle or narrower disc 672 thickness result in increased distributive mixing7,55-58. As suggested by the phase 673 diagram and also identified in previous extrusion trials Zone 1 is the region of the 674 extruder where there are melting of ibuprofen and xylitol, dissolution of IsoNA into 675 molten xylitol, and precipitation of cocrystal from the xylitol melt. Given the 676 complexity involved in this zone, an attempt was made to separate the nature of 677 mixing in Zone 1 . As Table 2 shows, two 0/2/10 sections were introduced to Zone 1 678 in SCP3 in order to provide additional dispersive mixing. These two half sections 679 were configured as shown in Figure 8c. The incorporation of such elements to the 680 first kneading region was to enhance dissolution of IsoNA in xylitol, a process that 681 was shown to be essential for cocrystallisation. It was considered that the dispersive 682 mixing sections might improve IsoNA-xylitol molecular dispersion as well as 683 facilitate Ibu-IsoNA molecular collision, hence improving cocrystallisation 684 efficiency ${ }^{59-62}$. Whilst in the second kneading region, the SCP3 employed an 685 additional 90/4/10 block in order to offer prolonged distributive mixing, thus 686 improving distribution of the cocrystal particulates suspended within the xylitol 687 matrix without further reducing the particle size and rendering them amorphous. 688 Further enhanced cocrystal yield was obtained with SCP3 at all extrusion TP 689 settings. In particular, TPs $1-4$ resulted in $19.9 \pm 3.1 \%, 18.1 \pm 0.9 \%, 35.0 \pm 2.9 \%$ and, $69058.8 \pm 1.8 \%$ cocrystal yielding, respectively, whilst TP5 resulted in a $39.8 \pm 1.7 \%$ yield. 691 All results show good agreement with our SCP design hypothesis and previous 692 discussion. 


\section{CONCLUSION}

694 In this study, we report for the first time the use of phase diagrams derived from F$695 \mathrm{H}$ theory in (1) determining miscibility behaviour; (2) selection of matrix carrier 696 and; (3) providing guidance on HME processing temperature for cocrystal ternary 697 systems. The use of small-scale thermal analysis and the adaptation of the Flory698 Huggins theory provide useful information such as binary solubility and correlation 699 between such solubility and temperature. This information was used in an attempt 700 to predict the conditions under which cocrystal yield could be significantly 701 improved. The construction of phase diagrams was useful in determining the HME 702 processing temperature at which cocrystal yield may be thermodynamically 703 favoured whereas screw profile experiments have shown that screw geometry 704 modification can also be employed to alter cocrystal yield. By optimum selection of 705 processing temperature and careful choice of extruder screw design, we have 706 obtained cocrystal yield of approximately $60 \%$ in the presence of a matrix carrier. It 707 is important to note that such a conversion ratio was achieved on a bench-top lab708 scale extrusion compounder. Through the use of larger processing equipment, 709 greater flexibility would be available in terms of both temperature setting and screw 710 profile across the barrel. This may allow for improved control and hence further 711 increased yield of cocrystal during scale-up. 
Table 1 Temperature profile settings TP1 TP5 designed to provide different thermodynamic environment for various degrees of mixing among raw ingredients.

\begin{tabular}{ccccc}
\hline & $\begin{array}{c}\text { Zone } \mathbf{0} \\
{ }^{\circ} \mathbf{C}\end{array}$ & $\begin{array}{c}\text { Zone } \mathbf{1} \\
{ }^{\circ} \mathbf{C}\end{array}$ & $\begin{array}{c}\text { Zone 2 } \\
{ }^{\circ} \mathbf{C}\end{array}$ & $\begin{array}{c}\text { Zone 3 } \\
{ }^{\circ} \mathbf{C}\end{array}$ \\
\hline TP1 & 60 & 80 & 80 & 80 \\
TP2 & 60 & 95 & 85 & 80 \\
TP3 & 60 & 100 & 90 & 80 \\
TP4 & 60 & 110 & 95 & 80 \\
TP5 & 60 & 115 & 100 & 80 \\
Extrusion & & & & \\
direction & & & & \\
\hline
\end{tabular}


Table 2 Specification of the screw configuration profile on one assembly shaft arranged from underneath the feed throat to the discharge end.

\begin{tabular}{|c|c|c|c|c|c|}
\hline \multirow{2}{*}{ No.* } & SCP1 & \multirow{2}{*}{ No. } & SCP2 & \multirow{2}{*}{ No. } & \multirow{2}{*}{$\begin{array}{c}\text { SCP3 } \\
\text { Element type }\end{array}$} \\
\hline & Element type & & Element type & & \\
\hline 19 & Forward conveying & 6 & Forward conveying & 6 & Forward conveying \\
\hline \multirow[t]{13}{*}{1} & End unit & 1 & $60^{\circ}$ kneading & 0.75 & $60^{\circ}$ kneading \\
\hline & & 1 & $90^{\circ}$ kneading & 0.5 & $0^{\circ}$ kneading \\
\hline & & 1 & $60^{\circ}$ kneading & 0.5 & $60^{\circ}$ kneading \\
\hline & Screw tip & 4 & Forward conveying & 0.5 & $0^{\circ}$ kneading \\
\hline & & 1 & $60^{\circ}$ kneading & 0.75 & $90^{\circ}$ kneading \\
\hline & & 1 & $90^{\circ}$ kneading & 1 & $60^{\circ}$ kneading \\
\hline & & 1 & $60^{\circ}$ kneading & 3 & Forward conveying \\
\hline & & 3 & Forward conveying & 1 & $60^{\circ}$ kneading \\
\hline & & 1 & End unit & 2 & $90^{\circ}$ kneading \\
\hline & & & Screw tip & 1 & $60^{\circ}$ kneading \\
\hline & & & & 2 & Forward conveying \\
\hline & & & & 1 & End unit \\
\hline & & & & & Screw tip \\
\hline
\end{tabular}

* Number of element unit (Length/Diameter). 
Table 3 Nomenclature and process conditions for the hot-melt extruded 1:1 Ibu-IsoNA cocrystal suspensions. Note that Ibu and IsoNA were premixed at a 1:1 molar ratio, whereas the weight fraction of xylitol in the ternary mixture was 50 wt $\%$ for all extruded formulations.

\begin{tabular}{|c|c|c|c|c|c|c|c|}
\hline \multirow{2}{*}{ Formulation } & \multirow{2}{*}{$\begin{array}{c}\text { Temperature } \\
\text { profile }\end{array}$} & \multirow{2}{*}{$\begin{array}{c}\text { Screw } \\
\text { configuration } \\
\text { profile }\end{array}$} & Zone 0 & Zone 1 & Zone 2 & Zone 3 & \multirow{2}{*}{$\begin{array}{l}\text { Cocrystal } \\
\text { Yield } \\
\quad(\%) \\
\end{array}$} \\
\hline & & & \multicolumn{4}{|c|}{ Actual recordings $\left({ }^{\circ} \mathrm{C}\right)$} & \\
\hline 1 & TP1 & & $60.3 \pm 0.28$ & $80.2 \pm 0.14$ & $80.0 \pm 0.07$ & $80.0 \pm 0.00$ & $9.0 \pm 0.2$ \\
\hline 2 & TP2 & & $60.4 \pm 0.64$ & $95.2 \pm 0.35$ & $87.8 \pm 1.20$ & $80.2 \pm 0.07$ & $9.7 \pm 1.2$ \\
\hline 3 & TP3 & SCP1 & $63.0 \pm 1.20$ & $100.2 \pm 0.28$ & $91.6 \pm 0.49$ & $82.6 \pm 0.57$ & $12.6 \pm 2.0$ \\
\hline 4 & TP4 & & $66.6 \pm 2.33$ & $110.2 \pm 0.21$ & $101.4 \pm 0.28$ & $87.9 \pm 0.57$ & $31.4 \pm 1.9$ \\
\hline 5 & TP5 & & $66.4 \pm 2.05$ & $115.4 \pm 0.14$ & $104.1 \pm 0.28$ & $89.2 \pm 0.71$ & $22.1 \pm 1.2$ \\
\hline 6 & TP1 & & $60.1 \pm 0.14$ & $80.2 \pm 0.07$ & $80.65 \pm 0.21$ & $80.0 \pm 0.07$ & $13.8 \pm 0.3$ \\
\hline 7 & TP2 & & $60.2 \pm 0.21$ & $95.1 \pm 0.14$ & $88.2 \pm 0.21$ & $80.3 \pm 0.14$ & $13.9 \pm 0.1$ \\
\hline 8 & TP3 & SCP2 & $61.6 \pm 0.49$ & $100.2 \pm 0.14$ & $93.2 \pm 0.28$ & $88.3 \pm 0.28$ & $20.6 \pm 0.4$ \\
\hline 9 & TP4 & & $63.0 \pm 0.57$ & $110.2 \pm 0.07$ & $105.3 \pm 0.57$ & $90.2 \pm 3.04$ & $44.2 \pm 0.3$ \\
\hline 10 & TP5 & & $65.9 \pm 0.14$ & $115.4 \pm 0.28$ & $107.0 \pm 0.14$ & $91.5 \pm 0.85$ & $30.5 \pm 0.8$ \\
\hline 11 & TP1 & & $60.0 \pm 0.07$ & $80.4 \pm 0.42$ & $81.1 \pm 0.28$ & $80.0 \pm 0.07$ & $19.9 \pm 3.1$ \\
\hline 12 & TP2 & & $60.0 \pm 0.00$ & $95.0 \pm 0.00$ & $87.8 \pm 0.99$ & $81.1 \pm 1.56$ & $18.1 \pm 0.9$ \\
\hline 13 & TP3 & SCP3 & $60.3 \pm 0.28$ & $100.2 \pm 0.28$ & $95.0 \pm 0.35$ & $86.0 \pm 2.69$ & $35.0 \pm 2.9$ \\
\hline 14 & TP4 & & $62.8 \pm 0.35$ & $110.0 \pm 0.07$ & $102.6 \pm 2.62$ & $89.0 \pm 4.17$ & $58.8 \pm 1.8$ \\
\hline 15 & TP5 & & $65.5 \pm 0.42$ & $115.1 \pm 0.14$ & $107.0 \pm 0.21$ & $90.8 \pm 2.55$ & $39.8 \pm 1.7$ \\
\hline
\end{tabular}



mpound structure and the calculated solubility parameters with component group contributions (group contributions indbook ${ }^{15}$.

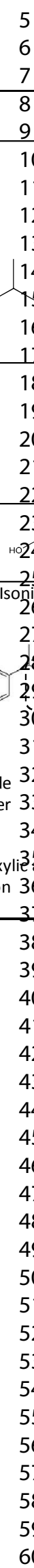

5

6

7

8

10

11

12

1

12

1

16

0 inamide

20

22 N

23 oH

$44-\mathrm{CH}_{2}-$

sonicotinamide equimolar cocrystal 26

27

$288^{-}-{ }^{-\cdots}$

29. Carboxylic acid-pyridine

$\left.30^{-}-7\right]$ synthon

3
3

vilie

n 36

37

38

39

40

41

42

43

44

45

46

47

48

49

50

51

52

53

54

55

56

57 
Table 5 The molecular weights, true densities and melting temperatures of each individual compound used in the Flory-Huggins calculations. The melting temperature shown here represents the mean \pm SD of three replicates. Note that the melting temperature of Isonicotinamide was determined at a heating rate of $200^{\circ} \mathrm{C} / \mathrm{min}$ to minimise influenced by sublimation.

\begin{tabular}{lcccccc}
\hline Compound & $\begin{array}{c}\mathbf{M}_{\mathbf{w}} \\
\mathbf{g} / \mathbf{m o l}\end{array}$ & $\begin{array}{c}\mathbf{\rho}^{*} \\
\mathbf{g} / \mathbf{c m}^{3}\end{array}$ & $\begin{array}{c}\mathbf{T}_{\mathbf{m} \text { (onset) }} \\
{ }^{\circ} \mathbf{C}\end{array}$ & $\begin{array}{c}\mathbf{T}_{\mathbf{m} \text { (peak) }} \\
{ }^{\circ} \mathbf{C}\end{array}$ & $\begin{array}{c}\mathbf{T}_{\text {m(end) }} \\
{ }^{\circ} \mathbf{C}\end{array}$ & $\begin{array}{c}\Delta \mathbf{H} \\
\mathbf{J} / \mathbf{g}\end{array}$ \\
\hline Ibuprofen & 206.30 & 1.08 & $75.37 \pm 0.22$ & $76.42 \pm 0.06$ & $78.86 \pm 0.08$ & $123.87 \pm 4.08$ \\
Isonicotinamide & 122.12 & 1.34 & $155.37 \pm 0.45$ & $162.58 \pm 0.97$ & $169.79 \pm 1.62$ & $199.21 \pm 9.80$ \\
Reference cocrystal & 656.48 & 1.18 & $120.21 \pm 0.26$ & $120.73 \pm 0.04$ & $120.96 \pm 0.06$ & $128.42 \pm 1.91$ \\
Xylitol & 152.15 & 1.52 & $93.95 \pm 0.31$ & $95.00 \pm 0.13$ & $95.32 \pm 0.14$ & $229.36 \pm 10.64$ \\
\hline
\end{tabular}

*The RSD for true density in the above measurements were all less than 0.07 . 


\section{Figure legends}

Figure 1 Schematic illustration of cocrystallisation mechanism, inside the extruder barrel, from a non-complementary carrier excipient.

Figure 2 DSC thermograms of (a) xylitol/lbu mixtures obtained using a heating rate of $1^{\circ} \mathrm{C} / \mathrm{min}$; (b) IsoNA/xylitol mixtures obtained using a heating rate of $200^{\circ} \mathrm{C} / \mathrm{min}$; and (c) reference cocrystal/xylitol mixtures obtained using a heating rate of $1^{\circ} \mathrm{C} / \mathrm{min}$ with various compositions.

Figure 3 The linear fit of the $F-H$ interaction parameter $\chi$ and $1 / T$ in Xylitol systems: $(A)$ Ibu-Xylitol; (B) Isonicotinamide-Xylitol; and (C) equimolar cocrystal-Xylitol blends, respectively.

Figure 4 A plot of $\Delta G_{\text {mix }} / R T$ versus composition for (a) Ibu/xylitol, (b) IsoNA/xylitol and (c) reference cocrystal/xylitol mixtures, respectively, at various temperatures: $\| 80^{\circ} \mathrm{C} ; \Delta 92^{\circ} \mathrm{C}$; $-95^{\circ} \mathrm{C} ; * 100^{\circ} \mathrm{C} ; 110^{\circ} \mathrm{C} ;+115^{\circ} \mathrm{C}$ and,$-120^{\circ} \mathrm{C}$.

Figure 5 Liquid-solid phase transition diagrams for (a) xylitol/ibuprofen, (b) IsoNA/xylitol and (c) ref-cocrystal/xylitol mixtures, respectively. The solid diamonds represent the average $\chi$ determined from $(n=3)$ replicate melting depression experiments, the dashed is the predicted solid-liquid line; the crosses give the F-H spinodal decomposition curve; and the solid triangle represents the critical solution temperature.

Figure 6 Overlain T- $\Phi$ spinodal lines for (from top down): cocrystal/xylitol, IsoNA/xylitol and, ibuprofen/xylitol systems, respectively. The arrows indicate regions in which respective phase behaviour (miscibility or separation) would occur based on the critical temperature predictions. It is clear that for the studied ternary system, an ideal HME processing window that meets all hypothesised criteria does not exist.

Figure 7 Calculated cocrystal yield from the melt-extruded suspensions. The pattern of the column represents the screw configuration profile: (blue) full-conveying profile SCP1, (green) intermediate intensity mixing profile SCP2, and (red) high intensity mixing profile SCP3, respectively. The label at the outer end of each column is the calculated average yield in percentage from 5 replicates. Note the columns are grouped according to the melting zone temperature setting.

Figure 8 Arrangement of: (a) forward conveying regions; (b) kneading regions used in SCP 2; (c) kneading region with two half-a-block-long $0^{\circ}$ dispersive mixing sections in SCP 3 and; (d) consecutive neutral kneading sections in SCP 3.

Figure 9 Illustrative sketches showing the distribution and dispersion of materials in the staggering kneading region of an intermeshing modular twin-screw extruder: (a) distributive mixing caused by forcing material to flow around the kneading discs of a 90/4/10 block; (b) dispersive mixing flow of a 60/4/10 block caused by forcing material to flow through the narrow gap between the top of the shearing disc tip and the interior wall of extruder barrel. 


\section{Table captions}

Table 1 Temperature profile settings TP1 TP5 designed to provide different thermodynamic environment for various degrees of mixing among raw ingredients.

Table 2 Specification of the screw configuration profile on one assembly shaft arranged from underneath the feed throat to the discharge end.

Table 3 Nomenclature and process conditions for the hot-melt extruded 1:1 lbu-IsoNA cocrystal suspensions. Note that Ibu and IsoNA were premixed at a 1:1 molar ratio, whereas the weight fraction of xylitol in the ternary mixture was $50 w t \%$ for all extruded formulations.

Table 4 Compound structure and the calculated solubility parameters with component group contributions (group contributions obtained from Polymer Handbook ${ }^{15}$.

Table 5 The molecular weights, true densities and melting temperatures of each individual compound used in the Flory-Huggins calculations. The melting temperature shown here represents the mean $\pm S D$ of three replicates. Note that the melting temperature of Isonicotinamide was determined at a heating rate of $200^{\circ} \mathrm{C} / \mathrm{min}$ to minimise influenced by sublimation. 


\section{Reference:}

(1) Li, S.; Yu, T.; Tian, Y.; McCoy, C. P.; Jones, D. S.; Andrews, G. P. Mechanochemical Synthesis of Pharmaceutical Cocrystal Suspensions via Hot Melt Extrusion: Feasibility Studies and Physicochemical Characterization. Mol. Pharm. 2016, 13 (9), 3054-3068.

(2) Bond, M.; Dunning, N. Xylitol. In Sweeteners and Sugar Alternatives in Food Technology; Blackwell Publishing Ltd, 2006; pp 295-328.

(3) Eddleston, M. D.; Patel, B.; Day, G. M.; Jones, W. Cocrystallization by FreezeDrying: Preparation of Novel Multicomponent Crystal Forms. Cryst. Growth Des. 2013, 13 (10), 4599-4606.

(4) Shan, N.; Toda, F.; Jones, W. Mechanochemistry and Co-Crystal Formation: Effect of Solvent on Reaction Kinetics. Chem. Commun. 2002, No. 20, 2372-2373.

(5) Dhumal, R.; Kelly, A.; York, P.; Coates, P.; Paradkar, A. Cocrystalization and Simultaneous Agglomeration Using Hot Melt Extrusion. Pharm. Res. 2010, 27 (12), 2725-2733.

(6) Rauwendaal, C. 10. Twin Screw Extruders. In Polymer Extrusion; Hanser: Munich, 2001; Vol. Revised 4t, pp 576-633.

(7) Giles, H. F.; Wagner, J. R.; Mount, E. M. Extrusion: The Definitive Processing Guide and Handbook; William Andrew Pub: Norwich, NY, 2005.

(8) Hancock, B. C.; York, P.; Rowe, R. C. The Use of Solubility Parameters in Pharmaceutical Dosage Form Design. Int. J. Pharm. 1997, 148 (1), 1-21.

(9) Greenhalgh, D. J.; Williams, A. C.; Timmins, P.; York, P. Solubility Parameters as Predictors of Miscibility in Solid Dispersions. J. Pharm. Sci. 1999, 88 (11), 11821190.

(10) Forster, A.; Hempenstall, J.; Tucker, I.; Rades, T. Selection of Excipients for Melt Extrusion with Two Poorly Water-Soluble Drugs by Solubility Parameter Calculation and Thermal Analysis. Int. J. Pharm. 2001, 226 (1-2), 147-161.

(11) Mohammad, M. A.; Alhalaweh, A.; Velaga, S. P. Hansen Solubility Parameters as a Tool to Predict Cocrystal Formation. Int. J. Pharm. 2011, 407, 63-71.

(12) Fukte, S. R.; Wagh, M. P.; Rawat, S. Coformer Selection: An Important Tool in Cocrystal Formation. Int. J. Pharm. Pharm. Sci. 2014, 6 (7), 9-14.

(13) Fedors, R. F. A Method for Estimating Both the Solubility Parameters and Molar Volumes of Liquids. Polym. Eng. Sci. 1974, 14 (2), 147-154. 
(14) Van Krevelen, D. W.; Hoftyzer, P. J. Properties of Polymers, Their Estimation and Correlation with Chemical Structure; Elsevier Scientific Pub. Co.: Amsterdam and New York, 1976; Vol. 2nd.

(15) Grulke, E. A. Solubility Parameter Values. In Polymer Handbook; Brandrup, J., Immergut, E. H., Grulke, E. A., Eds.; John Wiley \& Sons, Inc.: New York. Chichester. Weinheim. Brisbane. Singapore. Toronto., 1999; Vol. Fourth, p VII/675-VII/687.

(16) Donnelly, C.; Tian, Y.; Potter, C.; Jones, D. S.; Andrews, G. P. Probing the Effects of Experimental Conditions on the Character of Drug-Polymer Phase Diagrams Constructed Using Flory-Huggins Theory. Pharm. Res. 2015, 32 (1), 167-179.

(17) Padrela, L.; de Azevedo, E. G.; Velaga, S. P. Powder X-Ray Diffraction Method for the Quantification of Cocrystals in the Crystallization Mixture . Drug Dev. Ind. Pharm. 2012, 38 (8), 923-929.

(18) Guidelines, I. C. H. Internation Conference on Harmonization (ICH) Harmonized Tripartite Guideline, Note for Guidance on Validation of Analytical Procedures: Methodology. ICH Steering Committee 1996.

(19) Flory, P. J. Thermodynamics of High Polymer Solutions . J. Chem. Phys. 1941, 9 (8), 660.

(20) Flory, P. J. Thermodynamics of High Polymer Solutions. J. Chem. Phys. 1942, 10 (51).

(21) Huggins, M. L. Solutions of Long Chain Compounds . J. Chem. Phys. 1941, 9 (5), 440.

(22) Huggins, M. L. Some Properties of Solutions of Long-Chain Compounds. J. Phys. Chem. 1942, 46 (1), 151-158.

(23) Lin, D.; Huang, Y. A Thermal Analysis Method to Predict the Complete Phase Diagram of Drug-Polymer Solid Dispersions. Int. J. Pharm. 2010, 399, 109-115.

(24) Nishi, T.; Wang, T. T. Melting Point Depression and Kinetic Effects of Cooling on Crystallization in Poly(vinylidene Fluoride)-Poly(methyl Methacrylate) Mixtures. Macromolecules 1975, 8 (6), 909-915.

(25) Jo, W. H.; Kwan, I. H. Equation of State Theory for Melting Point Depression in Miscible Polymer Blends. Macromolecules 1991, 24 (11), 3368-3372.

(26) Marsac, P. J.; Li, T.; Taylor, L. S. Estimation of Drug-Polymer Miscibility and Solubility in Amorphous Solid Dispersions Using Experimentally Determined Interaction Parameters. Pharm. Res. 2009, 26 (1), 139-151. 
(27) Paudel, A.; Van Humbeeck, J.; Van Den Mooter, G. Theoretical and Experimental Investigation on the Solid Solubility and Miscibility of Naproxen in Poly(vinylpyrrolidone). Mol. Pharm. 2010, 7 (4), 1133-1148.

(28) Zhao, Y. Y.; Inbar, P.; Chokshi, H.; Malick, A. W.; Choi, D. S. Prediction of the Thermal Phase Diagram of Amorphous Solid Dispersions by Flory-Huggins Theory. J. Pharm. Sci. 2011, 100 (8), 3196-3207.

(29) Tian, Y. W.; Booth, J.; Meehan, E.; Jones, D. S.; Li, S.; Andrews, G. P. Construction of Drug-Polymer Thermodynamic Phase Diagrams Using Flory-Huggins Interaction Theory: Identifying the Relevance of Temperature and Drug Weight Fraction to Phase Separation within Solid Dispersions. Mol. Pharm. 2013, 10 (1), 236-248.

(30) Tian, Y.; Caron, V.; Jones, D. S.; Healy, A.-M.; Andrews, G. P. Using Flory-Huggins Phase Diagrams as a Pre-Formulation Tool for the Production of Amorphous Solid Dispersions: A Comparison between Hot-Melt Extrusion and Spray Drying. J. Pharm. Pharmacol. 2014, 66 (2), 256-274.

(31) Li, S.; Tian, Y.; Jones, D. S.; Andrews, G. P. Optimising Drug Solubilisation in Amorphous Polymer Dispersions: Rational Selection of Hot-Melt Extrusion Processing Parameters. AAPS PharmSciTech 2016, 17 (1), 200-213.

(32) Rubinstein, M.; Colby, R. Polymer Physics; Oxford University Press Inc.: New York, 2003.

(33) Almarsson, 0.; Zaworotko, M. J. Crystal Engineering of the Composition of Pharmaceutical Phases. Do Pharmaceutical Co-Crystals Represent a New Path to Improved Medicines? . Chem. Commun. 2004, 7 (17), 1889-1896.

(34) Basavoju, S.; Boström, D.; Velaga, S. Indomethacin-Saccharin Cocrystal: Design, Synthesis and Preliminary Pharmaceutical Characterization. Pharmaceutical Research. Springer Netherlands 2008, 25 (3), pp 530-541.

(35) Alhalaweh, A.; Velaga, S. P. Formation of Cocrystals from Stoichiometric Solutions of Incongruently Saturating Systems by Spray Drying. Cryst. Growth Des. 2010, 10 (8), 3302-3305.

(36) Prasad, R. V.; Rakesh, M. G.; Jyotsna, R. M.; Mangesh, T.; Anita, P. S.; Mayur, P. K. Pharmaceutical Cocrystallization: A Review. Int. J. Pharm. Chem. Sci. 2012, 1 (3), 725-736.

(37) Almarsson, Ö.; Peterson, M. L.; Zaworotko, M. J. The A to Z of Pharmaceutical Cocrystals: A Decade of Fast Moving New Science and Patents. Pharm. Pat. Anal. 
2012, 1 (3), 313-327.

(38) FriŠčić, T.; Trask, A. V; Jones, W.; Motherwell, W. D. S. Screening for Inclusion Compounds and Systematic Construction of Three-Component Solids by LiquidAssisted Grinding. Angew. Chemie 2006, 118 (45), 7708-7712.

(39) FriŠčić, T.; Fabian, L.; Burley, J. C.; Jones, W.; Motherwell, W. D. S. Exploring Cocrystal-Cocrystal Reactivity via Liquid-Assisted Grinding: Assembling of Racemic and Dismantling of Enantiomeric Cocrystals . Chem. Commun. 2006, 28 (48), 5009-5011.

(40) FriŠčić, T.; Childs, S. L.; Rizvi, S. A. A.; Jones, W. The Role of Solvent in Mechanochemical and Sonochemical Cocrystal Formation: A Solubility-Based Approach for Predicting Cocrystallisationoutcome. Cryst. Growth Des. 2009, 11 (3), 418-426.

(41) Lin, H.-L.; Hsu, P.-C.; Lin, S.-Y. Theophylline-citric Acid Co-Crystals Easily Induced by DSC-FTIR Microspectroscopy or Different Storage Conditions. Asian J. Pharm. Sci. 2013, 8 (1), 19-27.

(42) Shevchenko, A.; Miroshnyk, I.; Pietilä, L.-O.; Haarala, J.; Salmia, J.; Sinervo, K.; Mirza, S.; van Veen, B.; Kolehmainen, E.; Yliruusi, J. Diversity in Itraconazole Cocrystals with Aliphatic Dicarboxylic Acids of Varying Chain Length . Cryst. Growth Des. 2013, 13 (11), 4877-4884.

(43) Zhang, G. G. Z.; Henry, R. F.; Borchardt, T. B.; Lou, X. Efficient Co-Crystal Screening Using Solution-Mediated Phase Transformation. J. Pharm. Sci. 2007, 96 (5), 990995.

(44) Etter, M. C.; Reutzel, S. M.; Choo, C. G. Self-Organization of Adenine and Thymine in the Solid State. J. Am. Chem. Soc. 1993, 115 (10), 4411-4412.

(45) Trask, A. V.; Jones, W. Crystal Engineering of Organic Cocrystals by the Solid-State Grinding Approach. Topics in Current Chemistry. 2005, 254, pp 41-70.

(46) Liu, X.; Lu, M.; Guo, Z.; Huang, L.; Feng, X.; Wu, C. Improving the Chemical Stability of Amorphous Solid Dispersion with Cocrystal Technique by Hot Melt Extrusion . Pharm. Res. 2012, 29 (3), 806-817.

(47) Seefeldt, K.; Miller, J.; Alvarez-Núñez, F.; Rodríguez-Hornedo, N. Crystallization Pathways and Kinetics of Carbamazepine?nicotinamide Cocrystals from the Amorphous State by in Situ Thermomicroscopy, Spectroscopy, and Calorimetry Studies. J. Pharm. Sci. 2007, 96 (5), 1147-1158. 
(48) Hansen, C. M. Hansen Solubility Parameters: A User's Handbook; CRC Press: Boca Raton, 2007; Vol. Second.

(49) Abbott, S.; Hansen, C. M. Hansen Solubility Parameters in Practice; HansenSolubility.com, 2008.

(50) Yang, M.; Wang, P.; Gogos, C. G. A New Systematic Methodology to Determine Drug's Solubility in Polymer. In ANTEC; 2011.

(51) Aakeröy, C. B.; Beatty, A. M.; Helfrich, B. A.; Nieuwenhuyzen, M. Do Polymorphic Compounds Make Good Cocrystallizing Agents? A Structural Case Study That Demonstrates the Importance of Synthon Flexibility. Cryst. Growth Des. 2003, 3 (2), 159-165.

(52) Li, J.; Bourne, S. a; Caira, M. R. New Polymorphs of Isonicotinamide and Nicotinamide. Chem. Commun. 2011, 47 (5), 1530-1532.

(53) Berry, D. J.; Seaton, C. C.; Clegg, W.; Harrington, R. W.; Coles, S. J.; Horton, P. N.; Hursthouse, M. B.; Storey, R.; Jones, W.; Friscic, T.; Blagden, N. Applying Hot-Stage Microscopy to Co-Crystal Screening: A Study of Nicotinamide with Seven Active Pharmaceutical Ingredients . - Cryst. Growth Des. 2008, No. 5, 1697-1712.

(54) Rauwendaal, C. Mixing in Polymer Processing; M. Dekker: New York, 1991; Vol. 23.

(55) Nakayama, Y.; Takeda, E.; Shigeishi, T.; Tomiyama, H.; Kajiwara, T. Melt-Mixing by Novel Pitched-Tip Kneading Disks in a Co-Rotating Twin-Screw Extruder. Chem. Eng. Sci. 2011, 66 (1), 103-110.

(56) Yang, H.-H.; Manas-Zloczower, I. Flow Field Analysis of the Kneading Disc Region in a Co-Rotating Twin Screw Extruder. Polym. Eng. Sci. 1992, 32 (19), 14111417.

(57) Kalyon, D. M.; Sangani, H. N. An Experimental Study of Distributive Mixing in Fully Intermeshing, Co-Rotating Twin Screw Extruders. Polym. Eng. Sci. 1989, 29 (15), 1018-1026.

(58) Sarhangi Fard, A.; Anderson, P. D. Simulation of Distributive Mixing inside Mixing Elements of Co-Rotating Twin-Screw Extruders. Comput. Fluids 2013.

(59) Wang, W.; Manas-Zloczower, I. Analysis of Dispersive and Distributive Mixing In Terms of Minor Component Size and Spatial Distributions in Continuous Polymer Processing Equipment. 2000.

(60) Paul, E. L.; Atiemo-Obeng, V.; Kresta, S. M. Handbook of Industrial Mixing: Science and Practice; Jon Wiley \& Sons, Inc.: New Jersy, 2004. 
(61) Manas-Zloczower, I. Mixing and Compounding of Polymers: Theory and Practice; Hanser, 2009.

(62) Gogos, C. G.; Liu, H.; Wang, P. Laminar Dispersive and Distributive Mixing with Dissolution and Applications to Hot-Melt Extrusion. In Hot-Melt Extrusion: Pharmaceutical Applications; Douroumis, D., Ed.; John Wiley \& Sons, Ltd, 2012; pp 261-284. 
Figure 1 Schematic illustration of cocrystallisation mechanism, inside the extruder barrel, from a noncomplementary carrier excipient.

$70 \times 33 \mathrm{~mm}(300 \times 300 \mathrm{DPI})$ 

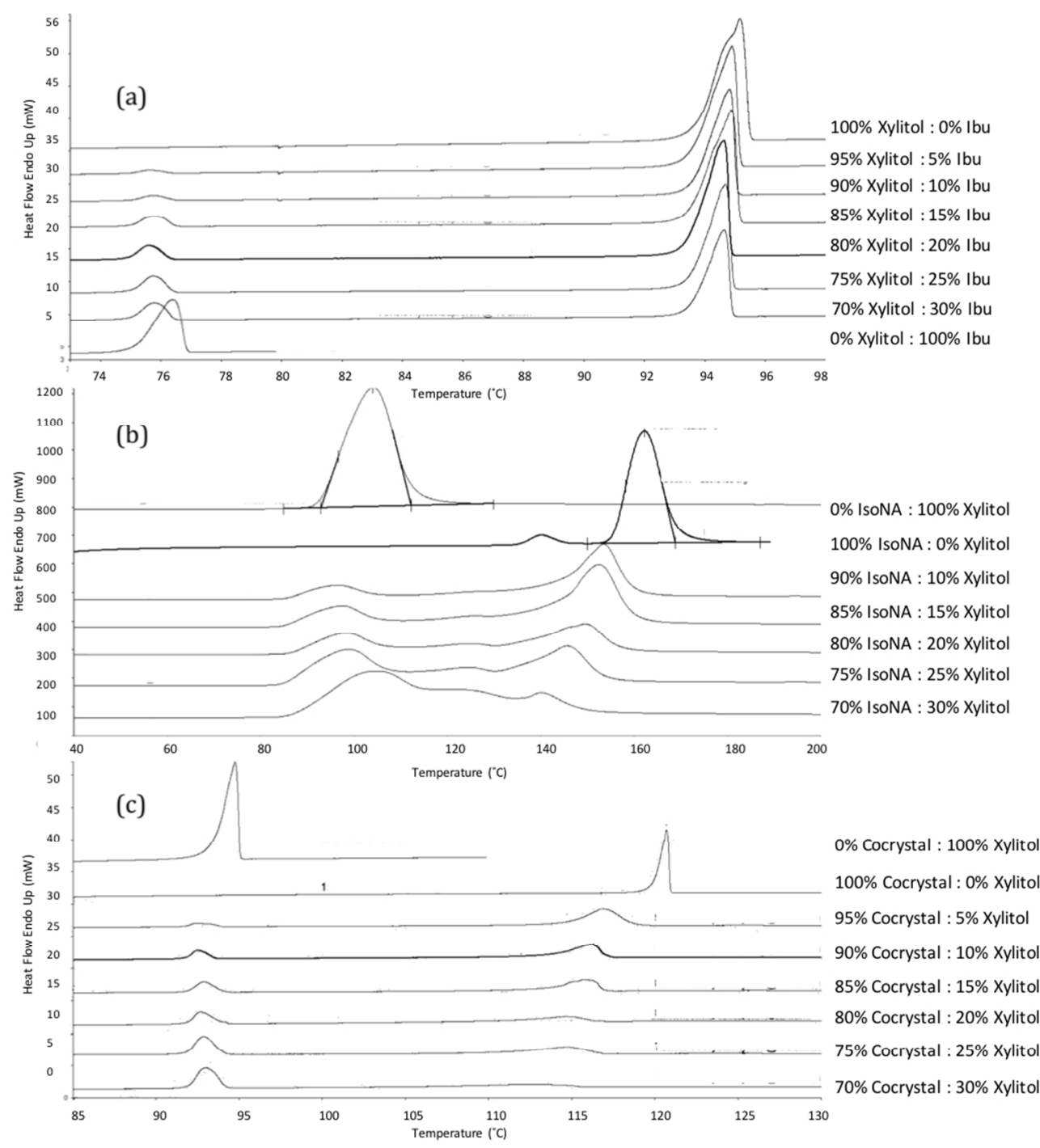

Figure 2 DSC thermograms of (a) xylitol/Ibu mixtures obtained using a heating rate of $1^{\circ} \mathrm{C} / \mathrm{min}$; (b) IsoNA/xylitol mixtures obtained using a heating rate of $200^{\circ} \mathrm{C} / \mathrm{min}$; and (c) reference cocrystal/xylitol mixtures obtained using a heating rate of $1^{\circ} \mathrm{C} / \mathrm{min}$ with various compositions.

\section{$175 \times 190 \mathrm{~mm}(150 \times 150 \mathrm{DPI})$}



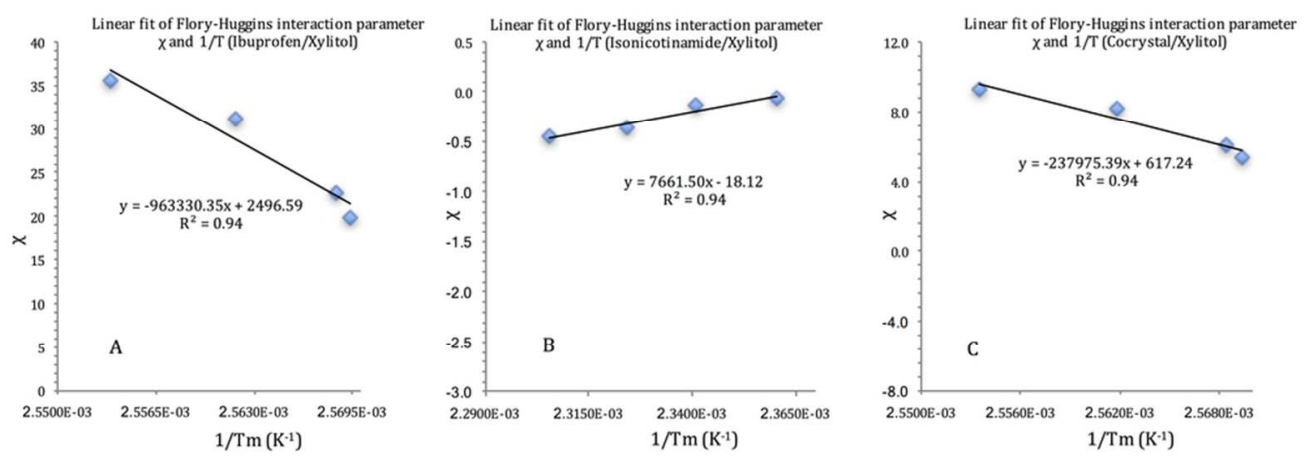

Figure 3 The linear fit of the $F-H$ interaction parameter $X$ and 1/T in Xylitol systems: (A) Ibu-Xylitol; (B) Isonicotinamide-Xylitol; and (C) equimolar cocrystal-Xylitol blends, respectively.

$84 \times 29 \mathrm{~mm}(300 \times 300$ DPI $)$ 

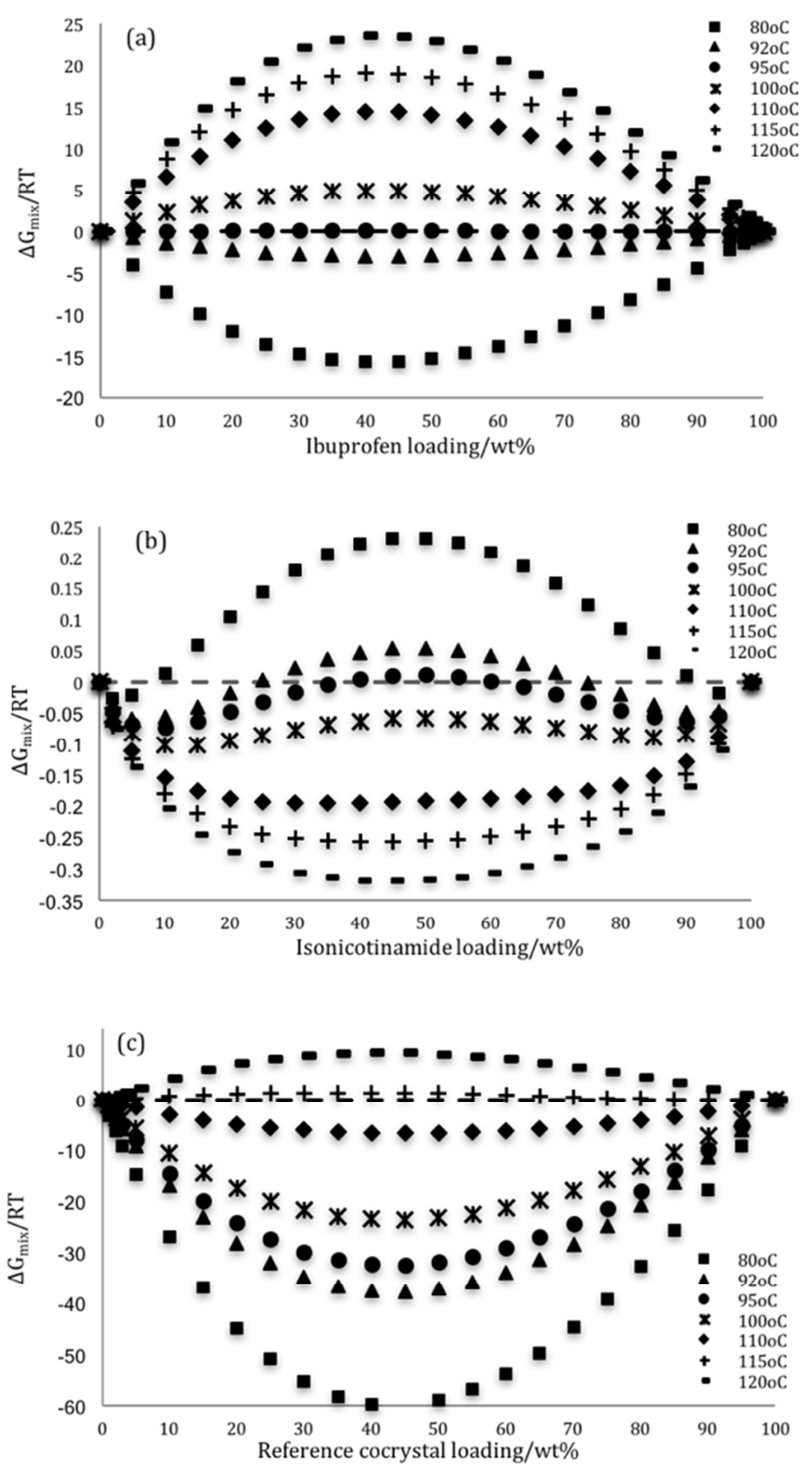

Figure $4 \mathrm{~A}$ plot of $\Delta \mathrm{Gmix} / \mathrm{RT}$ versus composition for (a) Ibu/xylitol, (b) IsoNA/xylitol and (c) reference cocrystal/xylitol mixtures, respectively, at various temperatures: $80^{\circ} \mathrm{C} ; 92^{\circ} \mathrm{C} ; 95^{\circ} \mathrm{C} ; 100^{\circ} \mathrm{C} ; 110^{\circ} \mathrm{C} ; 115^{\circ} \mathrm{C}$ and, $120^{\circ} \mathrm{C}$, respectively.

$107 \times 190 \mathrm{~mm}(150 \times 150 \mathrm{DPI})$ 

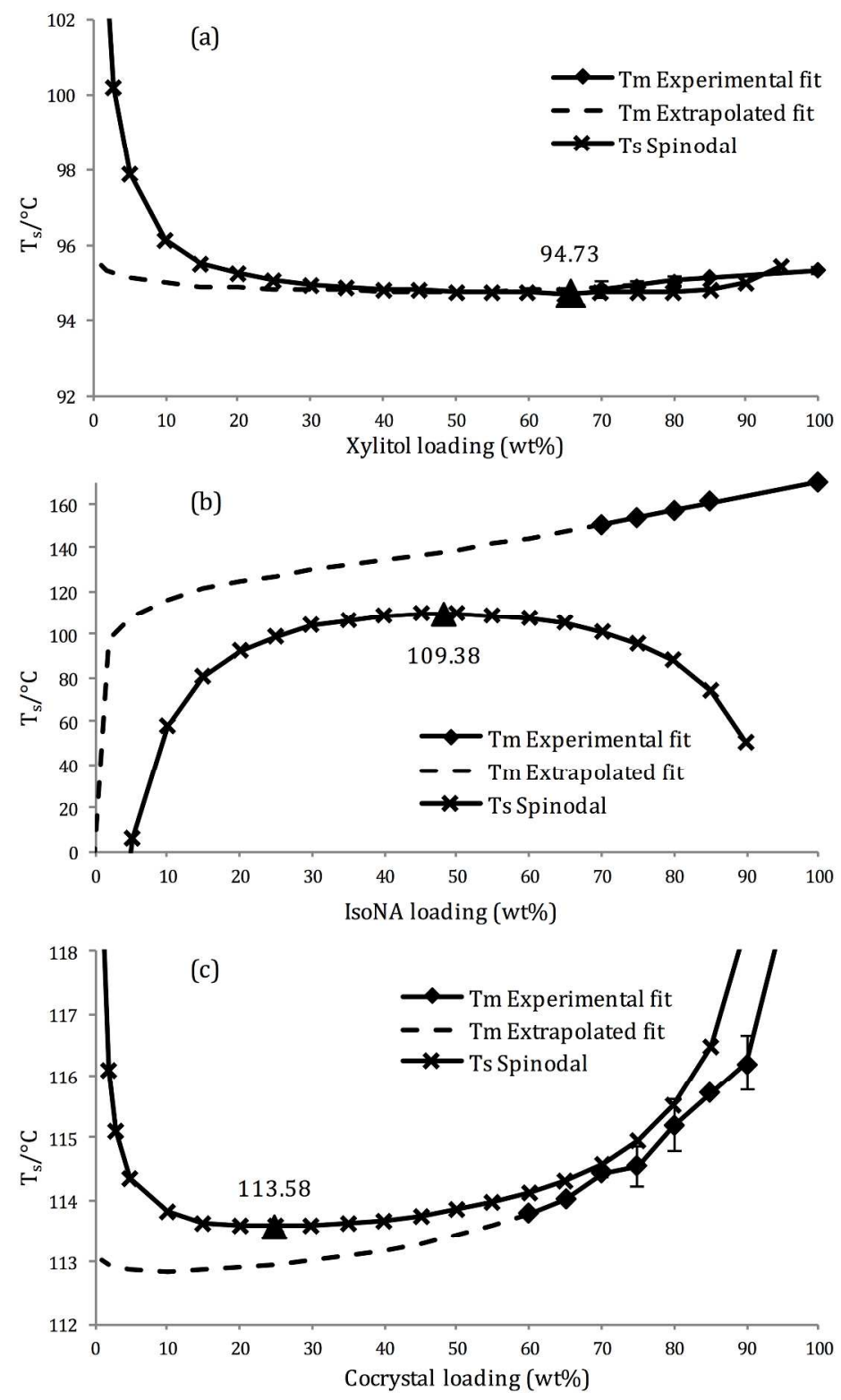

Figure 5 Liquid-solid phase transition diagrams for (a) xylitol/ibuprofen, (b) IsoNA/xylitol and (c) refcocrystal/xylitol mixtures, respectively. The solid diamonds represent the average $x$ determined from $(n=3)$ replicate melting depression experiments, the dashed is the predicted solid-liquid line; the crosses give the

$\mathrm{F}-\mathrm{H}$ spinodal decomposition curve; and the solid triangle represents the critical solution temperature.

$$
209 \times 346 \mathrm{~mm}(300 \times 300 \text { DPI })
$$


Figure 6 Overlain T- $\Phi$ spinodal lines for (from top down): cocrystal/xylitol, IsoNA/xylitol and, ibuprofen/xylitol systems, respectively. The arrows indicate regions in which respective phase behaviour (miscibility or separation) would occur based on the critical temperature predictions. It is clear that for the studied ternary system, an ideal HME processing window that meets all hypothesised criteria does not exist.

$118 \times 45 \mathrm{~mm}(300 \times 300 \mathrm{DPI})$ 


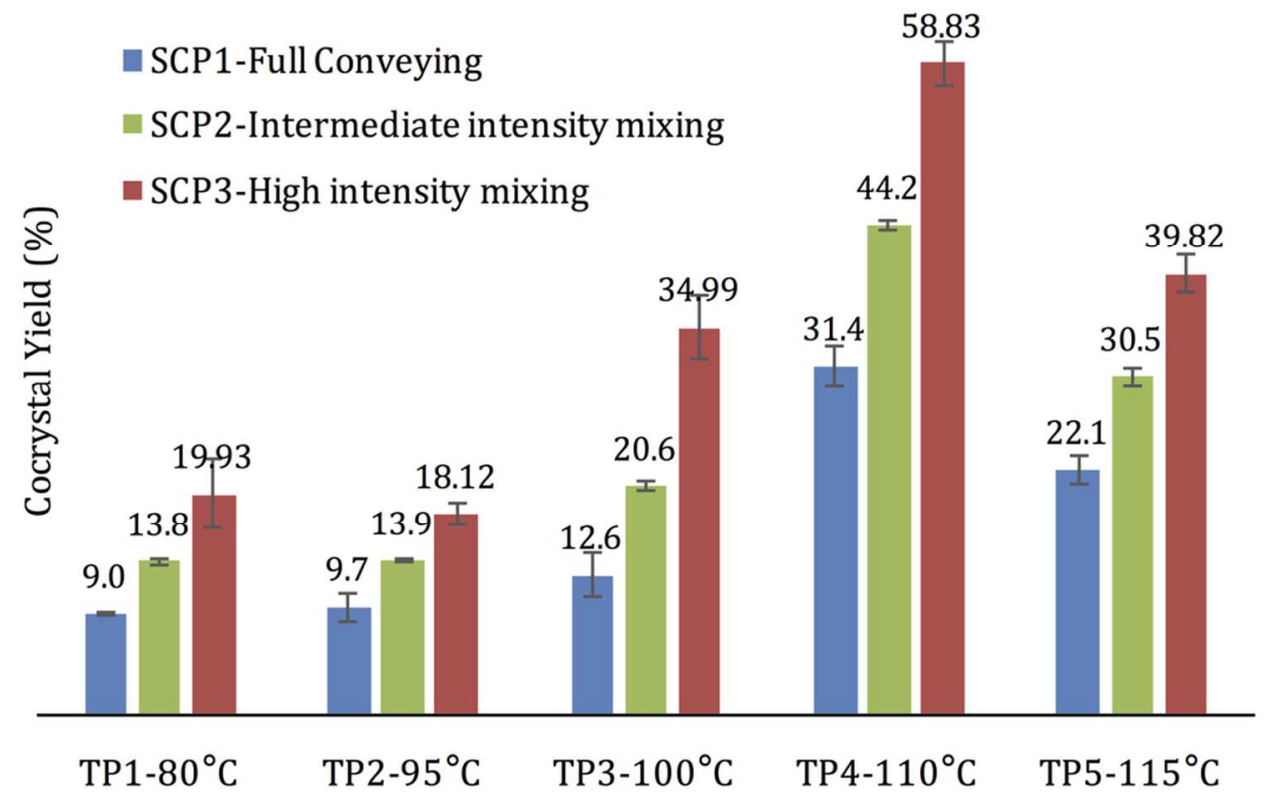

Figure 7 Calculated cocrystal yield from the melt-extruded suspensions. The pattern of the column represents the screw configuration profile: (blue) full-conveying profile SCP1, (green) intermediate intensity mixing profile SCP2, and (red) high intensity mixing profile SCP3, respectively. The label at the outer end of each column is the calculated average yield in percentage from 5 replicates. Note the columns are grouped according to the melting zone temperature setting.

$93 \times 59 \mathrm{~mm}(300 \times 300 \mathrm{DPI})$ 


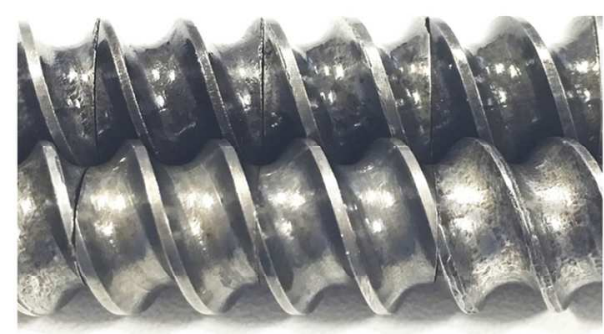

a.

Flow direction

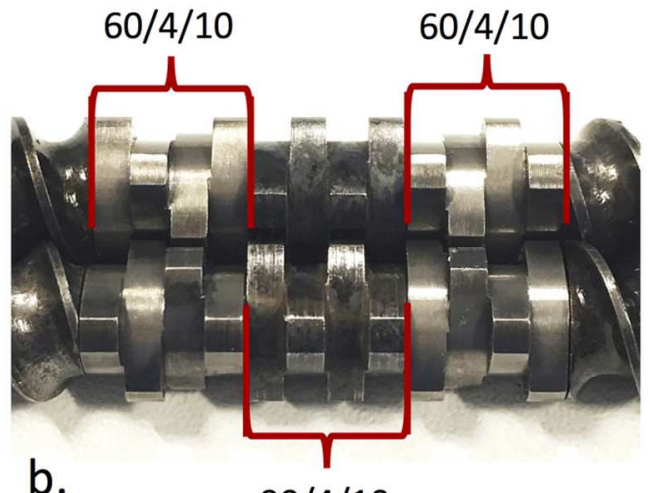

b.

$90 / 4 / 10$
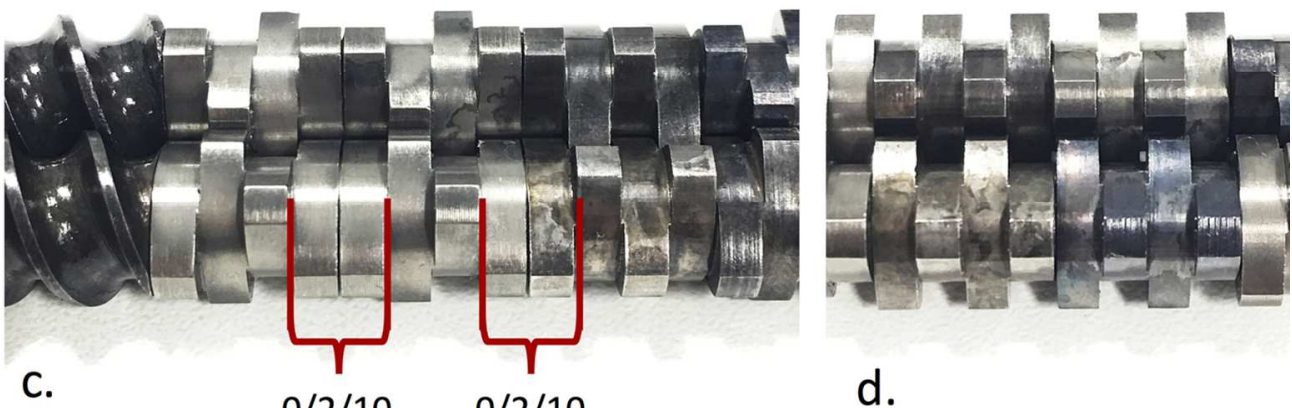

d.

Figure 8 Arrangement of: (a) forward conveying regions; (b) kneading regions used in SCP 2; (c) kneading region with two half-a-block-long $0^{\circ}$ dispersive mixing sections in SCP 3 and; (d) consecutive neutral kneading sections in SCP 3.

$116 \times 93 \mathrm{~mm}(300 \times 300 \mathrm{DPI})$ 

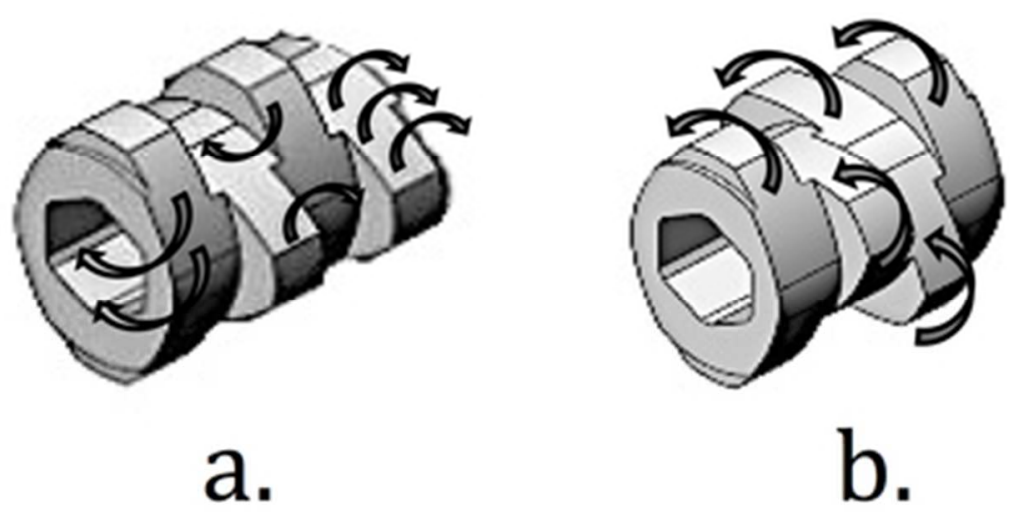

Figure 9 Illustrative sketches showing the distribution and dispersion of materials in the staggering kneading region of an intermeshing modular twin-screw extruder: (a) distributive mixing caused by forcing material to flow around the kneading discs of a 90/4/10 block; (b) dispersive mixing flow of a 60/4/10 block caused by forcing material to flow through the narrow gap between the top of the shearing disc tip and the interior wall of extruder barrel.

$35 \times 18 \mathrm{~mm}(300 \times 300 \mathrm{DPI})$ 


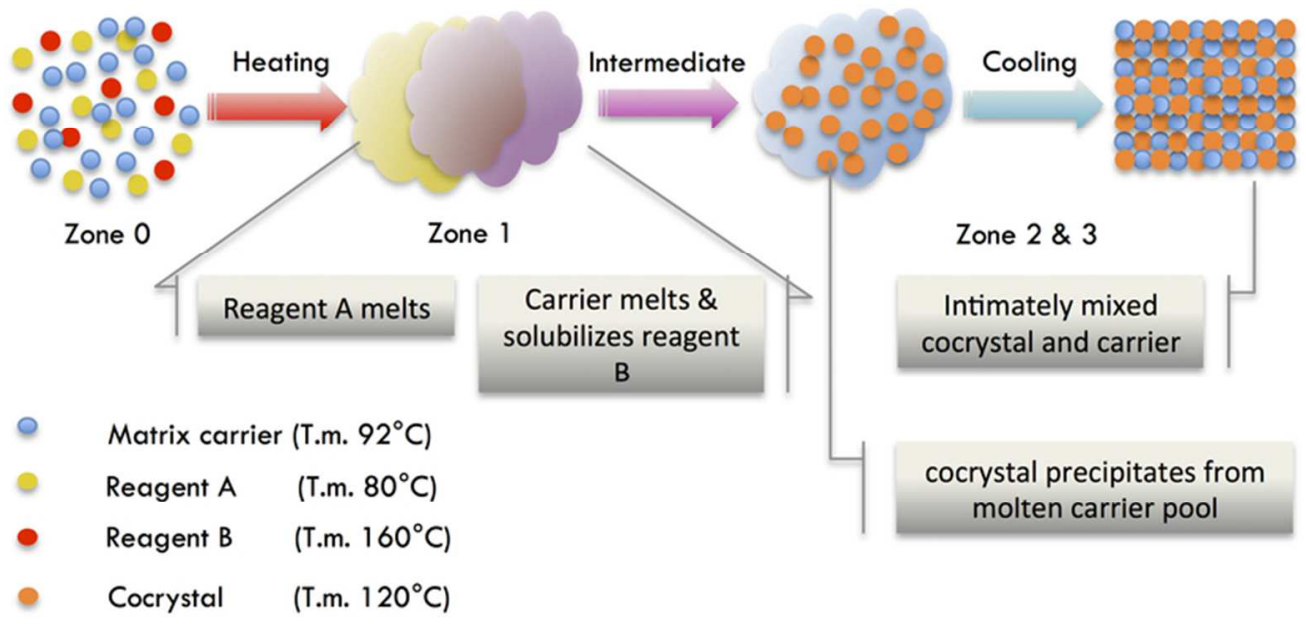

Table of Contents Graphic

$70 \times 33 \mathrm{~mm}(300 \times 300$ DPI $)$ 